\title{
DESCRIPTION OF UNITARY REPRESENTATIONS OF THE GROUP OF INFINITE $p$-ADIC INTEGER MATRICES
}

\author{
YURY A. NERETIN
}

\begin{abstract}
We classify irreducible unitary representations of the group of all infinite matrices over a $p$-adic field $(p \neq 2)$ with integer elements equipped with a natural topology. Any irreducible representation passes through a group GL of infinite matrices over a residue ring modulo $p^{k}$. Irreducible representations of the latter group are induced from finite-dimensional representations of certain open subgroups.
\end{abstract}

\section{INTRODUCTION}

\subsection{Notations and definitions.}

(a) Rings. Let $p$ be a prime,

$$
p>2 \text {. }
$$

Let $\mathbb{Z}_{p^{n}}:=\mathbb{Z} / p^{n} \mathbb{Z}$ be a residue ring, $\mathbb{F}_{p}:=\mathbb{Z}_{p}$ be the field with $p$ elements. The ring of $p$-adic integers $\mathbb{O}_{p}$ is the projective limit

$$
\mathbb{O}_{p}=\lim _{\longleftarrow n} \mathbb{Z}_{p^{n}}
$$

of the following chain (see, e.g., 32]):

$$
\cdots \longleftarrow \mathbb{Z}_{p^{n-1}} \longleftarrow \mathbb{Z}_{p^{n}} \longleftarrow \mathbb{Z}_{p^{n+1}} \longleftarrow \ldots,
$$

we have $\mathbb{Z}_{p^{n}}=\mathbb{O}_{p} / p^{n} \mathbb{O}_{p}$. Denote by $\mathbb{Q}_{p}$ the field of $p$-adic numbers.

(b) The infinite Symmetric Group AND Oligomorphic Groups. Let $\Omega$ be a countable set. Denote by $S(\Omega)$ the group of all permutations of $\Omega$; denote $S_{\infty}:=$ $S(\mathbb{N})$. The topology on the infinite symmetric group $S(\Omega)$ is determined by the condition: stabilizers of finite subsets are open subgroups and these subgroups form a fundamental system of neighborhoods of the unit 1 Equivalently, a sequence $g^{(\alpha)}$ converges to $g$ if for each $\omega \in \Omega$ we have $\omega g^{(\alpha)}=\omega g$ for sufficiently large $\alpha$.

A closed subgroup $G$ of $S(\Omega)$ is called oligomorphic if for each $k$ it has only a finite number of orbits on the product $\Omega \times \cdots \times \Omega$ of $k$ copies of $\Omega$; see [5].

Received by the editors September 22, 2019, and, in revised form, April 14, 2021.

2020 Mathematics Subject Classification. Primary 22E50; Secondary 22E66, 20M18, 18 B99.

This work was supported by the grants FWF, P28421, P31591.

${ }^{1}$ Thus we get a structure of a Polish group. Moreover this topology is a unique separable topology on the infinite symmetric group; see [13. In particular, this means that a unitary representation of $S_{\infty}$ in a separable Hilbert space is automatically continuous. 
(c) Modules $\mathfrak{l}\left(\mathbb{Z}_{p^{n}}\right)$ AND Groups $\operatorname{GL}\left(\infty, \mathbb{Z}_{p^{n}}\right)$. Define the module $\mathfrak{l}\left(\mathbb{Z}_{p^{n}}\right)$ as the set of all sequences $v=\left(v_{1}, v_{2}, \ldots\right)$, where $v_{j} \in \mathbb{Z}_{p^{n}}$ and $v_{j}=0$ for sufficiently large $j$. The set $\mathfrak{l}\left(\mathbb{Z}_{p^{n}}\right)$ is countable; we equip it with a discrete topology. Denote by $e_{j}$ the standard basis elements, i.e., $e_{j}$ has a unit on $j$-th place, other elements are 0 .

Define groups $\mathrm{GL}\left(\infty, \mathbb{Z}_{p^{n}}\right)$ as groups of infinite invertible matrices $g$ over $\mathbb{Z}_{p^{n}}$ such that:

- each row of $g$ contains only a finite number of nonzero elements;

- each column contains only a finite number of nonzero elements;

- the inverse matrix $g^{-1}$ satisfies the same conditions.

Notice that rows of a matrix $g$ are precisely vectors $e_{i} g$, and columns are $e_{j} g^{t}$ (we denote by $g^{t}$ a transposed matrix).

Actually, the topic of this paper is representations of $\mathrm{GL}\left(\infty, \mathbb{Z}_{p^{n}}\right)$.

This group is continual and we must define a topology on $\mathrm{GL}\left(\infty, \mathbb{Z}_{p^{n}}\right)$. A sequence $g^{(\alpha)} \in \mathrm{GL}\left(\infty, \mathbb{Z}_{p^{n}}\right)$ converges to $g$ if all sequences $e_{i} g^{(\alpha)}$ and $e_{i}\left(g^{(\alpha)}\right)^{t}$ are eventually constant and their limits are $e_{i} g$ and $e_{j} g^{t}$ respectively. Thus we get a structure of a totally disconnected topological group.

The group $\mathrm{GL}\left(\infty, \mathbb{Z}_{p^{n}}\right)$ acts on the countable set $\mathfrak{l}\left(\mathbb{Z}_{p^{n}}\right) \oplus \mathfrak{l}\left(\mathbb{Z}_{p^{n}}\right)$ by transformations

$$
(v, w) \mapsto\left(v g, w\left(g^{t}\right)^{-1}\right) .
$$

In particular, this defines an embedding of $\mathrm{GL}\left(\infty, \mathbb{Z}_{p^{n}}\right)$ to a symmetric group $S\left(\mathfrak{l}\left(\mathbb{Z}_{p^{n}}\right) \oplus \mathfrak{l}\left(\mathbb{Z}_{p^{n}}\right)\right)$. The image of the group $\mathrm{GL}\left(\infty, \mathbb{Z}_{p^{n}}\right)$ is a closed subgroup of $S\left(\mathfrak{l}\left(\mathbb{Z}_{p^{n}}\right) \oplus \mathfrak{l}\left(\mathbb{Z}_{p^{n}}\right)\right)$ and the induced topology coincides with the natural topology on $\operatorname{GL}\left(\infty, \mathbb{Z}_{p^{n}}\right)$. By [27, Lemma 3.7], the group $\operatorname{GL}\left(\infty, \mathbb{Z}_{p^{n}}\right)$ is oligomorphic.

(d) Modules $\mathfrak{l}\left(\mathbb{O}_{p}\right)$ And Groups $\mathrm{GL}\left(\infty, \mathbb{O}_{p}\right)$. Denote by $\mathfrak{l}\left(\mathbb{O}_{p}\right)$ the set of all sequences $r=\left(r_{1}, r_{2}, \ldots\right)$, where $r_{j} \in \mathbb{O}_{p}$ and $\left|r_{j}\right| \rightarrow 0$ as $j \rightarrow \infty$. The space $\mathfrak{l}\left(\mathbb{O}_{p}\right)$ is a projective limit,

$$
\mathfrak{l}\left(\mathbb{O}_{p}\right)=\lim _{\longleftarrow} \mathfrak{l}\left(\mathbb{Z}_{p^{n}}\right),
$$

we equip it with the topology of the projective limit. In other words, a sequence $r^{(j)} \in \mathfrak{l}\left(\mathbb{O}_{p}\right)$ converges if for any $p^{n}$ the reduction of $r^{(j)}$ modulo $p^{n}$ is eventually constant in $\mathbb{Z}_{p^{n}}$.

We define $\operatorname{GL}\left(\infty, \mathbb{O}_{p}\right)$ as the group of all infinite matrices $g$ over $\mathbb{O}_{p}$ such that:

- each row of $g$ is an element of $\mathfrak{l}\left(\mathbb{O}_{p}\right)$;

- each column of $g$ is an element of $\mathfrak{l}\left(\mathbb{O}_{p}\right)$;

- the matrix $g$ has an inverse and $g^{-1}$ satisfies the same conditions.

We say that a sequence $g^{(\alpha)} \in \mathrm{GL}\left(\infty, \mathbb{O}_{p}\right)$ converges to $g$ if for any $i$ the sequence $e_{i} g^{(\alpha)}$ converges to $e_{i} g$ and for any $j$ the sequence $e_{i}\left(g^{(\alpha)}\right)^{t}$ converges to $e_{j} g^{t}$. This determines a structure of a totally disconnected topological group on $\operatorname{GL}\left(\infty, \mathbb{O}_{p}\right)$.

We have obvious homomorphisms $\mathrm{GL}\left(\infty, \mathbb{Z}_{p^{n}}\right) \rightarrow \mathrm{GL}\left(\infty, \mathbb{Z}_{p^{n-1}}\right)$, the group $\mathrm{GL}\left(\infty, \mathbb{O}_{p}\right)$ is the projective limit

$$
\operatorname{GL}\left(\infty, \mathbb{O}_{p}\right)=\lim _{\longleftarrow n} \operatorname{GL}\left(\infty, \mathbb{Z}_{p^{n}}\right)
$$

and its topology is the topology of projective limit. 
1.2. Preliminary remarks. A priori we know the following statement:

\section{Theorem 1.1.}

(a) The group $\mathrm{GL}\left(\infty, \mathbb{O}_{p}\right)$ is a type I group; it has a countable number of irreducible unitary representations. Any unitary representation $\mathrm{GL}\left(\infty, \mathbb{O}_{p}\right)$ is a sum of irreducible representations. Any irreducible unitary representation of $\mathrm{GL}\left(\infty, \mathbb{O}_{p}\right)$ is in fact a representation of some group $\mathrm{GL}\left(\infty, \mathbb{Z}_{p^{n}}\right)$.

(b) Each irreducible representation of $\mathrm{GL}\left(\infty, \mathbb{Z}_{p^{n}}\right)$ is induced from a finite-dimensional representation of an open subgroup. More precisely, for any irreducible unitary representation of $\mathrm{GL}\left(\infty, \mathbb{Z}_{p^{n}}\right)$ there exists an open subgroup $\widehat{Q} \subset \mathrm{GL}\left(\infty, \mathbb{Z}_{p^{n}}\right)$, a normal subgroup $Q \subset \widehat{Q}$ of finite index and an irreducible representation $\nu$ of $\widehat{Q}$, which is trivial on $Q$, such that $\rho$ is induced from $\nu$.

This is a special case of a theorem of Tsankov about unitary representations of oligomorphic groups and projective limits of holomorphic groups; see 34, Theorem 1.3] 2 It seems that 34, 2 are not sufficient to give a precise answer in our case.

Let us give a definition of an induced representation (see, e.g., [33, Sect. 7] and [15. Sect. 13]) which is appropriate in our case. Let $G$ be a totally disconnected separable group, $Q$ its open subgroup. Let $\nu$ be a unitary representation of $Q$ in a Hilbert space $V$. Consider the space $H$ of $V$-valued functions $f$ on a countable homogeneous space $Q \backslash G$ such that

$$
\sum_{x \in Q \backslash G}\|f(x)\|^{2}<\infty .
$$

Equip $H$ with the inner product

$$
\left\langle f_{1}, f_{2}\right\rangle_{H}:=\sum_{x \in Q \backslash G}\left\langle f_{1}(x), f_{2}(x)\right\rangle_{V} .
$$

Let $U$ be a function on $G \times(Q \backslash G)$ taking values in the group of unitary operators in $V$ such that:

- Formula

$$
\rho(g) f(x)=U(g, x) f(x g)
$$

determines a representation of $G$ in $H$.

- Let $x_{0}$ be the initial point of $Q \backslash G$, i.e., $x_{0} Q=x_{0}$. Then for $q \in Q$ we have $U\left(q, x_{0}\right)=\nu(q)$.

The first condition implies that the function $U(g, x)$ satisfies the functional equation

$$
U\left(x, g_{1} g_{2}\right)=U\left(x, g_{1}\right) U\left(x g_{1}, g_{2}\right) .
$$

It can be shown that $U(g, x)$ is uniquely defined up to a natural calibration

$$
U(g, x) \sim A(g x)^{-1} U(g, x) A(x),
$$

where $A$ is a function on $Q \backslash G$ taking values in the unitary group of $V$ (see, e.g., [15. Sect 13.1]). For this reason, an induced representation $\rho(g)=\operatorname{Ind}_{Q}^{G}(\nu)$ is canonically defined up to a unitary equivalence.

\footnotetext{
${ }^{2} \mathrm{~A}$ reduction of representations of $\mathrm{GL}\left(\infty, \mathbb{O}_{p}\right)$ to representations of quotients $\mathrm{GL}\left(\infty, \mathbb{Z}_{p^{\mu}}\right)$ easily follows from [20, Proposition VII.1.3]; see [27, Corollary 3.5]. In our proof of Theorem 1.5 Tsankov's theorem is used in the proof of Proposition 2.1] which was done in [27.
} 
We also can choose $U(g, x)$ in the following way. For any $x \in Q \backslash G$ we choose an element $s(x) \in G$ such that $x_{0} s(x)=x$. Then $U(g, x)=\nu(q)$, where $q$ is determined from the condition $s(x) g=q s(x g)$.

1.3. The statement. The result of the paper is Theorem 1.5, which claims that irreducible representations of $\mathbb{G}$ are induced from finite dimensional representations of certain family of subgroups $\mathbb{G}^{\circ}[L ; M]$; these subgroups are described in Lemma 1.3 .

Thus we fix a ring $\mathbb{Z}_{p^{\mu}}$ and examine the group

$$
\mathbb{G}:=\mathrm{GL}\left(\infty, \mathbb{Z}_{p^{\mu}}\right) \text {. }
$$

We consider two right actions of $\mathbb{G}$ on $\mathfrak{l}\left(\mathbb{Z}_{p^{\mu}}\right), g: v \mapsto v g, g: v \mapsto v\left(g^{t}\right)^{-1}$. Define a pairing

$$
\mathfrak{l}\left(\mathbb{Z}_{p^{\mu}}\right) \times \mathfrak{l}\left(\mathbb{Z}_{p^{\mu}}\right) \rightarrow \mathbb{Z}_{p^{\mu}}
$$

by

$$
\{v, w\}:=\sum v_{j} w_{j}=v w^{t},
$$

our action preserves this pairing, i.e.,

$$
\left\{v g, v\left(g^{t}\right)^{-1}\right\}=\{v, w\} .
$$

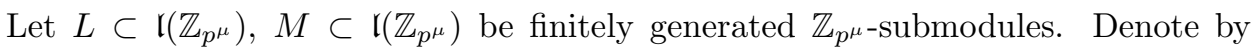
$\widehat{\mathbb{G}}[L ; M]$ the subgroup of $\mathbb{G}$ consisting of $g$ such that $L g=L$ and $M\left(g^{t}\right)^{-1}=M$. By $\mathbb{G}^{\circ}[L ; M] \subset \widehat{\mathbb{G}}[L ; M]$ we denote group of matrices fixing $L$ and $M$ pointwise. Obviously, the quotient group $\widehat{\mathbb{G}}[L ; M] / \mathbb{G}^{\circ}[L ; M]$ is finite; it acts on the direct sum $L \oplus M$ preserving the pairing $\{f, g\}$. Any irreducible representation $\tau$ of $\widehat{\mathbb{G}}[L ; M] / \mathbb{G}^{\circ}[L ; M]$ can be regarded as a representation $\widehat{\tau}$ the group $\widehat{\mathbb{G}}[L ; M]$, which is trivial on $\mathbb{G}^{\circ}[L ; M]$. For given $L, M, \tau$ we consider the representation

$$
\operatorname{Ind}_{\widehat{\mathbb{G}}[L ; M]}^{\mathbb{G}}(\widehat{\tau})
$$

of $\mathbb{G}$ induced from the representation $\widehat{\tau}$ of the group $\widehat{\mathbb{G}}[L ; M]$. Ol'shanski 30 ] obtained the following statement 3 for the group $\operatorname{GL}\left(\infty, \mathbb{F}_{p}\right)=\operatorname{GL}\left(\infty, \mathbb{Z}_{p}\right)$.

\section{Theorem 1.2.}

(a) Any irreducible unitary representation of the group $\mathrm{GL}\left(\infty, \mathbb{F}_{p}\right)$ has this form.

(b) Two irreducible representations can be equivalent only for a trivial reason, i.e.,

$$
\operatorname{Ind} \underset{\widetilde{G}\left[L_{1} ; M_{1}\right]}{\mathbb{G}}\left(\tau_{1}\right) \sim \operatorname{Ind}_{\widetilde{\mathbb{G}}\left[L_{2} ; M_{2}\right]}^{\mathbb{G}}\left(\tau_{2}\right)
$$

if and only if there exists $h \in \mathbb{G}$ such that $L_{1} h=L_{2} h, M_{1}\left(h^{t}\right)^{-1}=M_{2}$ and $\tau_{2}(q)=\tau_{1}\left(h q h^{-1}\right)$.

For groups $\mathrm{GL}\left(\infty, \mathbb{Z}_{p^{\mu}}\right)$ with $\mu>1$ the situation is more delicate. Let $L, M$ actually be contained in $\left(\mathbb{Z}_{p^{\mu}}\right)^{m} \subset \mathfrak{l}\left(\mathbb{Z}_{p^{\mu}}\right)$. Fix a matrix $b$ such that $\tan b=L$ and a matrix $c$ such that $\operatorname{ker} c^{t}=M$.

\footnotetext{
${ }^{3} \mathrm{~A}$ proof in [30] is only sketched; other proofs were given by Dudko [8] and Tsankov [34].

${ }^{4}$ We assume that each row of $b$ and each column of $c$ contain only a finite number of nonzero elements.
} 
Lemma 1.3. The group $\mathbb{G}^{\circ}[L ; M]$ consists of all invertible matrices admitting the following representation as a block matrix of size $m+\infty$ :

$$
g=\left(\begin{array}{cc}
a & b v \\
w c & z
\end{array}\right)
$$

where the block ' $a$ ' can be written in both forms

$$
a=1-b S, \quad a=1-T c .
$$

Next, define a subgroup $\mathbb{G}^{\bullet}[L ; M] \subset \mathbb{G}^{\circ}[L ; M]$ consisting of matrices having the form

$$
g=\left(\begin{array}{cc}
1-b u c & b v \\
w c & z
\end{array}\right)
$$

Proposition 1.4. The group $\mathbb{G}^{\bullet}[L ; M]$ is the minimal subgroup of finite index in $\widehat{\mathbb{G}}[L ; M]$, i.e., it is contained in any subgroup of finite index in $\widehat{\mathbb{G}}[L ; M]$.

\section{Theorem 1.5.}

(a) Any irreducible unitary representation of $\mathbb{G}$ is induced from a representation $\tau$ of some group $\widehat{\mathbb{G}}[L ; M]$ that is trivial on the subgroup $\mathbb{G} \bullet[L ; M]$.

(b) Two irreducible representations of this kind can be equivalent only for the trivial reason as in Theorem 1.2 .

Remark. Recall that $p \neq 2$. In several places of our proof we divide elements of residue rings $\mathbb{Z}_{p^{\mu}}$ by 2 . Usually, this division can be replaced by longer considerations. But in Lemma 6.8 this seems crucial.

Remark. Let $L, M \subset p \cdot \mathfrak{l}\left(\mathbb{Z}_{p^{\mu}}\right)$. Then $\mathbb{G}[L ; M]$ contains a congruence subgroup $N$ consisting of elements of $\mathbb{G}$ that are equal 1 modulo $p^{\mu-1}$. Since $N$ is a normal subgroup in $\mathbb{G}$, it is normal in $\widehat{\mathbb{G}}[L ; M]$. Let $\tau$ be trivial on $N$. Then the induced representation $\operatorname{Ind}_{\mathbb{G}[L ; M]}^{\mathbb{G}}(\widehat{\tau})$ is trivial on the congruence subgroup $N$ and actually we get representations of $\mathrm{GL}\left(\infty, \mathbb{Z}_{p^{\mu-1}}\right)$.

Remark. The statement (b) is a general fact for oligomorphic groups; see [34, Proposition 4.1(ii)]. So we omit a proof (in our case this can be easily established by examination of intertwining operators).

1.4. Remarks: Infinite-dimensional $p$-adic groups. Now there exists a welldeveloped representation theory of infinite symmetric groups and of infinite-dimensional real classical groups. Parallel development in the $p$-adic case meets some difficulties. However, infinite dimensional $p$-adic groups were a topic of sporadic attacks since late 1980s; see [19], 36, 18. We indicate some works on $p$-adic groups and their parallels with nontrivial constructions for real and symmetric groups.

(a) An extension of the Weil representation of the infinite-dimensional symplectic group $\operatorname{Sp}(2 \infty, \mathbb{C})$ to the semigroup of lattices (Nazarov [19, 18]; see a partial exposition in [22, Sect. 11.1-11.2]).

(b) A construction of projective limits of $p$-adic Grassmannians and quasiinvariant actions of $p$-adic GL $(\infty)$ on these Grassmannians [24. This is an analog of virtual permutations (or Chinese restaurant process, see, e.g., [1, 11.19]; they are a base of harmonic analysis related to infinite symmetric group, see [14]), and of projective limits of compact symmetric spaces (see [31, 21]); they are a standpoint for a harmonic analysis related to infinite-dimensional classical groups; see [3]. 
(c) An attempt to describe a multiplication of double cosets (see the next section) for $p$-adic classical groups in 25. In any case this leads to a strange geometric construction, namely to simplicial maps of Bruhat-Tits buildings whose boundary values are rational maps of $p$-adic Grassmannians.

(d) The work [4 contains a $p$-adic construction in the spirit of exchangeability 5 namely, descriptions of invariant ergodic measures on spaces of infinite $p$-adic matrices. By the Wigner-Mackey trick (see, e.g., [15, Sect. 13.3]), such kind of statements can be translated to a description of spherical functions on certain groups.

So during last years new elements of a nontrivial picture related to infinitedimensional $p$-adic groups appeared. For this reason, understanding of representations $\mathrm{GL}\left(\infty, \mathbb{O}_{p}\right)$ becomes necessary.

1.5. Another completion of a group of infinite matrices over $\mathbb{Z}_{p^{n}}$. Define a group $\mathcal{G}$ consisting of infinite matrices $g$ over $\mathbb{Z}_{p^{n}}$ such that:

- $g$ contains only a finite number of elements in each column;

- $g^{-1}$ exists and satisfies the same property.

A sequence $g^{(\alpha)}$ converges to $g$ if for each $j$ we have a convergence of $e_{j} g^{(\alpha)}$.

Clearly, $\mathcal{G} \supset \mathbb{G}$. Classification of irreducible unitary representations of $\mathcal{G}$ is the following. For each finitely generated submodule in $\mathfrak{l}\left(\mathbb{Z}_{p^{n}}\right)$ we consider the subgroup $\widehat{\mathcal{G}}[L]$ consisting of transformations sending $L$ to itself and the subgroup $\mathcal{G}^{\circ}[L]$ fixing $L$ pointwise.

Proposition 1.6. Any irreducible unitary representation of $\mathcal{G}$ is induced from a representation of some group $\widehat{\mathcal{G}}[L]$ trivial on $\mathcal{G}^{\circ}[L]$.

This follows from Theorem 1.5. on the other hand this can be deduced in a straightforward way from Tsankov's result 34 .

\section{Preliminaries: The Category of Double cosets}

2.1. Multiplication of double cosets and the category $\mathcal{K}$. Here we discuss a version of a general construction of multiplication of double cosets (see [29, 30, [20], [26], 27]).

Denote by $\mathbb{G}_{\text {fin }} \subset \mathbb{G}$ the subgroup of finitary matrices, i.e., matrices $g$ such that $g-1$ has only a finite number of nonzero elements. For $\alpha=0,1, \ldots$ denote by $\mathbb{G}(\alpha) \subset \mathbb{G}$ the subgroups consisting of matrices having the form $\left(\begin{array}{cc}1_{\alpha} & 0 \\ 0 & u\end{array}\right)$, where $1_{\alpha}$ denotes the unit matrix of size $\alpha$ and $u$ is an arbitrary invertible matrix over $\mathbb{Z}_{p^{\mu}}$. Obviously, $\mathbb{G}(\alpha)$ is isomorphic to $\mathbb{G}$. Consider double coset spaces $\mathbb{G}(\alpha) \backslash \mathbb{G} / \mathbb{G}(\beta)$; their elements are matrices determined up to the equivalence

$$
\left(\begin{array}{ll}
a & b \\
c & d
\end{array}\right) \sim\left(\begin{array}{cc}
1_{\alpha} & 0 \\
0 & u
\end{array}\right)\left(\begin{array}{ll}
a & b \\
c & d
\end{array}\right)\left(\begin{array}{cc}
1_{\beta} & 0 \\
0 & v
\end{array}\right)=\left(\begin{array}{cc}
a & b v \\
u c & u d v
\end{array}\right),
$$

where a matrix $g$ is represented as a block matrix of size $(\alpha+\infty) \times(\beta+\infty)$. For a matrix $g$ we write the corresponding double coset as

$$
\left[\begin{array}{l|l}
a & b \\
\hline c & d
\end{array}\right]_{\alpha \beta}
$$

\footnotetext{
${ }^{5}$ i.e., of higher analogs of the de Finetti theorem; see 1
} 
we will omit subscript $\alpha \beta$ if it is not necessary to indicate a size. We wish to define a natural multiplication

$$
\mathbb{G}(\alpha) \backslash \mathbb{G} / \mathbb{G}(\beta) \times \mathbb{G}(\beta) \backslash \mathbb{G} / \mathbb{G}(\gamma) \rightarrow \mathbb{G}(\alpha) \backslash \mathbb{G} / \mathbb{G}(\gamma) .
$$

Let $\mathfrak{g}_{1} \in \mathbb{G}(\alpha) \backslash \mathbb{G} / \mathbb{G}(\beta), \mathfrak{g}_{2} \in \mathbb{G}(\beta) \backslash \mathbb{G} / \mathbb{G}(\gamma)$ be double cosets. By [27, Lemma 4.1 , any double coset has a representative in $\mathbb{G}_{\mathrm{fin}}$. Choose such representatives $g_{1}$ and $g_{2}$ for $\mathfrak{g}_{1}, \mathfrak{g}_{2}$,

$$
g_{1}=\left[\begin{array}{l|ll}
a & b & \\
\hline c & d & \\
& & 1_{\infty}
\end{array}\right]_{\alpha \beta}, g_{1}=\left[\begin{array}{l|ll}
p & q & \\
\hline r & t & \\
& & 1_{\infty}
\end{array}\right]_{\beta \gamma} .
$$

Let sizes of submatrices $\left(\begin{array}{ll}a & b \\ c & d\end{array}\right),\left(\begin{array}{ll}p & q \\ r & t\end{array}\right)$, be $N \times N$. Denote by $\theta^{\beta}(j)$ the following matrix

$$
\theta^{\beta}(j):=\left(\begin{array}{c|ccc}
1_{\beta} & & & \\
\hline & 0 & 1_{j} & \\
& 1_{j} & 0 & \\
& & & 1_{\infty}
\end{array}\right) \in \mathbb{G}(\beta) .
$$

Consider the sequence

$$
\mathbb{G}(\alpha) \cdot g_{1} \theta^{\beta}(j) g_{2} \cdot \mathbb{G}(\gamma) \in \mathbb{G}(\alpha) \backslash \mathbb{G} / \mathbb{G}(\gamma) .
$$

It is more or less obvious that this sequence is eventually constant and its limit is

$$
\begin{aligned}
& \mathfrak{g}_{1} \circ \mathfrak{g}_{2}= \\
& =\left[\left(\begin{array}{c|ccc}
a & b & & \\
\hline c & d & & \\
& & 1_{L} & \\
& & & 1_{\infty}
\end{array}\right)\left(\begin{array}{c|ccc|cc}
1_{\beta} & & & \\
\hline & 0 & 1_{L} & \\
& 1_{L} & 0 & \\
& & & 1_{\infty}
\end{array}\right)\left(\begin{array}{c|ccc}
p & q & & \\
\hline r & t & & \\
& & 1_{L} & \\
& & & 1_{\infty}
\end{array}\right)\right]_{\alpha \gamma},
\end{aligned}
$$

where $L \geqslant N-\beta$. The final expression is

$$
\mathfrak{g}_{1} \circ \mathfrak{g}_{2}=\left[\begin{array}{c|ccc}
a p & a q & b & \\
\hline c p & c q & d & \\
r & t & 0 & \\
& & & 1_{\infty}
\end{array}\right]_{\alpha \gamma} \sim\left[\begin{array}{c|ccc}
a p & b & a q & \\
\hline c p & d & c q & \\
r & 0 & t & \\
& & & 1_{\infty}
\end{array}\right]_{\alpha \gamma} .
$$

In calculations below we use the last expression for o-product.

It is easy to verify that this multiplication is associative, i.e., for any

$$
\mathfrak{g}_{1} \in \mathbb{G}(\alpha) \backslash \mathbb{G} / \mathbb{G}(\beta), \quad \mathfrak{g}_{2} \in \mathbb{G}(\beta) \backslash \mathbb{G} / \mathbb{G}(\gamma), \quad \mathfrak{g}_{3} \in \mathbb{G}(\gamma) \backslash \mathbb{G} / \mathbb{G}(\delta),
$$

we have

$$
\left(\mathfrak{g}_{1} \circ \mathfrak{g}_{2}\right) \circ \mathfrak{g}_{3}=\mathfrak{g}_{1} \circ\left(\mathfrak{g}_{2} \circ \mathfrak{g}_{3}\right) .
$$

In other words, we get a category. Objects of this category are numbers $\alpha=$ $0,1,2, \ldots$. Sets of morphisms are

$$
\operatorname{Mor}(\beta, \alpha):=\mathbb{G}(\alpha) \backslash \mathbb{G} / \mathbb{G}(\beta) .
$$

The multiplication is given by formula (2.4). Denote this category by $\mathcal{K}$. 
The group of automorphisms $\operatorname{Aut}_{\mathcal{K}}(\alpha)$ is $\operatorname{GL}\left(\alpha, \mathbb{Z}_{p^{\mu}}\right)$; it consists of double cosets of the form $\left[\begin{array}{c|c}a & 0 \\ \hline 0 & 1_{\infty}\end{array}\right]$.

Next, the map $g \mapsto g^{-1}$ induces maps

$$
\mathbb{G}(\alpha) \backslash \mathbb{G} / \mathbb{G}(\beta) \rightarrow \mathbb{G}(\beta) \backslash \mathbb{G} / \mathbb{G}(\alpha),
$$

denote these maps by $\mathfrak{g} \mapsto \mathfrak{g}^{*}$. It is easy to see that we get an involution in the category $\mathcal{K}$, i.e.,

$$
\left(\mathfrak{g}_{1} \circ \mathfrak{g}_{2}\right)^{*}=\mathfrak{g}_{2}^{*} \circ \mathfrak{g}_{1}^{*} .
$$

The map $g \mapsto\left(g^{t}\right)^{-1}$ determines an automorphism of the category $\mathcal{K}$; denote it by $\mathfrak{g} \mapsto \mathfrak{g}^{\star}$. It sends objects to themselves and

$$
\left(\mathfrak{g}_{1} \circ \mathfrak{g}_{2}\right)^{\star}=\mathfrak{g}_{2}^{\star} \circ \mathfrak{g}_{1}^{\star} .
$$

Remarks on notation.

(1) In formulas (2.2), (2.3), (2.4), the last columns, the last rows, and the blocks $1_{\infty}$ contain no information and only enlarge sizes of matrices. For this reason, below we will omit them. Precisely, for a matrix $\left(\begin{array}{ll}a & b \\ c & d\end{array}\right)$ of finite size we denote

$$
\left[\begin{array}{c|c}
a & b \\
\hline c & d_{\star}
\end{array}\right]:=\left[\begin{array}{c|cc}
a & b & 0 \\
\hline c & d & 0 \\
0 & 0 & 1_{\infty}
\end{array}\right] \quad\left(\begin{array}{c|c}
a & b \\
\hline c & d_{\star}
\end{array}\right):=\left(\begin{array}{c|cc}
a & b & 0 \\
\hline c & d & 0 \\
0 & 0 & 1_{\infty}
\end{array}\right) .
$$

(2) We will denote a multiplication of $[g]$ by an automorphism $A$ as $A \cdot[g]$,

$$
\begin{aligned}
& A \cdot\left[\begin{array}{c|c}
a & b \\
\hline c & d_{\star}
\end{array}\right]:=\left[\begin{array}{c|c}
A & 0 \\
\hline 0 & 1
\end{array}\right] \circ\left[\begin{array}{c|c}
a & b \\
\hline c & d_{\star}
\end{array}\right]=\left[\begin{array}{c|c}
A a & A b \\
\hline c & d_{\star}
\end{array}\right] ; \\
& {\left[\begin{array}{c|c}
a & b \\
\hline c & d_{\star}
\end{array}\right] \cdot A^{\prime}:=\left[\begin{array}{c|c}
a & b \\
\hline c & d_{\star}
\end{array}\right] \circ\left[\begin{array}{c|c}
A^{\prime} & 0 \\
\hline 0 & 1
\end{array}\right]=\left[\begin{array}{c|c}
a A^{\prime} & b \\
\hline c A^{\prime} & d_{\star}
\end{array}\right] .}
\end{aligned}
$$

2.2. The multiplicativity theorem. Consider a unitary representation $\rho$ of the group $\mathbb{G}$ in a Hilbert space $H$. Denote by $H_{\alpha} \subset H$ the space of $\mathbb{G}(\alpha)$-fixed vectors. Denote by $P_{\alpha}$ the operator of orthogonal projection to $H_{\alpha}$.

\section{Proposition 2.1.}

(a) For any $\beta$ the sequence $\rho\left(\theta^{\beta}(j)\right)$ converges to $P_{\beta}$ in the weak operator topology.

(b) The space $\cup H_{\alpha}$ is dense in $H$.

The first statement is Lemma 1.1 from [27; the claim (b) is a special case of Proposition VII.1.3 from [20].

Let $g \in \mathbb{G}, \alpha, \beta \in \mathbb{Z}_{+}$. Consider the operator

$$
\widetilde{\rho}_{\alpha \beta}(g): H_{\beta} \rightarrow H_{\alpha}
$$

given by

$$
\widetilde{\rho}_{\alpha \beta}(g):=\left.P_{\alpha} \rho(g)\right|_{H_{\beta}} .
$$

It is easy to see that for $h_{1} \in \mathbb{G}(\alpha), h_{2} \in \mathbb{G}(\beta)$ we have

$$
\tilde{\rho}_{\alpha \beta}(g)=\widetilde{\rho}_{\alpha \beta}\left(h_{1} g h_{2}\right),
$$

i.e., $\widetilde{\rho}_{\alpha \beta}(g)$ actually depends on the double coset $\mathfrak{g}$ containing $g$. 


\section{Theorem 2.2.}

(a) The map $g \mapsto \widetilde{\rho}_{\alpha \beta}(\mathfrak{g})$ is a representation of the category $\mathcal{K}$, i.e., for any $\alpha$, $\beta$, $\gamma$ for any $\mathfrak{g}_{1} \in \operatorname{Mor}(\beta, \alpha), \mathfrak{g}_{2} \in \operatorname{Mor}(\gamma, \beta)$ we have

$$
\widetilde{\rho}_{\alpha \beta}\left(\mathfrak{g}_{1}\right) \widetilde{\rho}_{\beta \gamma}\left(\mathfrak{g}_{2}\right)=\widetilde{\rho}_{\alpha \gamma}\left(\mathfrak{g}_{1} \circ \mathfrak{g}_{2}\right) .
$$

(b) $\widetilde{\rho}$ is a*-representation, i.e.,

$$
\widetilde{\rho}_{\alpha \beta}(\mathfrak{g})^{*}=\widetilde{\rho}_{\beta \alpha}\left(\mathfrak{g}^{*}\right) .
$$

The statement (a) is an automatic corollary of Proposition 2.1] see [27, Theorem 2.1]. The statement (b) is obvious.

Remark. The considerations of Subsections 2.1, 2.2 are one-to-one repetitions of similar statements for real classical groups and symmetric groups; see [30, 28, [23], 26]. Further considerations drastically differ from these theories.

2.3. Structure of the paper. We derive the classification of unitary representations of $\mathbb{G}$ from the multiplicativity theorem and the following argumentation. The semigroups $\Gamma(m):=\operatorname{End}_{\mathcal{K}}(m)$ are finite. It is known that a finite semigroup with an involution has a faithful $*$-representation in a Hilbert space if and only if it is an inverse semigroup (see discussion below, Subsection 3.3). More generally, if a category having finite sets of morphisms acts faithfully in Hilbert spaces, then it must be an inverse category; see [12. However, semigroups $\operatorname{End}_{\mathcal{K}}(\alpha)$ are not inverse 6 and $*$-representations of $\mathcal{K}$ pass through a smaller category.

Section 3 contains preliminary remarks on inverse semigroup and construction of an inverse category $\mathcal{L}$, which is a quotient of $\mathcal{K}$. This provides us lower estimate of maximal inverse semigroup quotients of semigroups $\Gamma(m)$.

In Section 4 we examine idempotents in maximal inverse semigroup quotients $\operatorname{inv}(\Gamma(m))$ of $\Gamma(m)$. In Section 5 we show that some of idempotents of $\operatorname{inv}(\Gamma(m))$ act by the same operators in all representations of $\mathbb{G}$. Next, for any representation of $\mathbb{G}$ there is a minimal $m$ such that $H_{m} \neq 0$. In Section 6 we examine the image of $\Gamma(m)$ in such representation.

In Section 7 we discuss properties of the groups $\mathbb{G}^{\circ}[L ; M]$ and $\mathbb{G}^{\bullet}[L ; M]$.

The final part of the proof is contained in Section 8 .

\section{The REDUCED CATEGORY AND INVERSE SEMigRoups}

3.1. Notation. Below we work only with the group $\mathbb{G}:=\mathrm{GL}\left(\infty, \mathbb{Z}_{p^{\mu}}\right)$. To simplify notation, we write

$$
\operatorname{GL}(m):=\operatorname{GL}\left(m, \mathbb{Z}_{p^{\mu}}\right), \quad \Gamma(m):=\operatorname{End}_{\mathcal{K}}(m), \quad \mathfrak{l}^{m}:=\left(\mathbb{Z}_{p^{\mu}}\right)^{m} .
$$

For a unitary representation $\rho$ of a $\mathbb{G}$ we define the height $h(\rho)$ as the minimum of $\alpha$ such that $H_{\alpha} \neq 0$.

By $x(\bmod p)$ we denote a reduction of an object (a scalar, a vector, a matrix) defined over $\mathbb{Z}_{p^{\mu}}$ modulo $p$, i.e. to the field $\mathbb{F}_{p}$. Notice that a square matrix $A$ of finite size over $\mathbb{Z}_{p^{\mu}}$ is invertible if and only if $A(\bmod p)$ is invertible. A matrix $B$ is nilpotent (i.e., $B^{N}=0$ for sufficiently large $\left.N\right)$ if and only if $B(\bmod p)$ is nilpotent. Indeed, if $B^{k}=0(\bmod p)$, then $B^{k}$ has the form $p C$ for some matrix $C$. Hence, $\left(B^{k}\right)^{\mu+1}=p^{\mu+1} C^{\mu+1}=0$.

\footnotetext{
${ }^{6}$ This was observed by Ol'shanskǐ 30 for $\operatorname{GL}\left(\infty, \mathbb{F}_{p}\right)$.
} 
We use several symbols for equivalences in $\operatorname{Mor}_{\mathcal{K}}(\beta, \alpha)$; the $\sim$ was defined by (2.1); the symbols

$$
\equiv, \quad \approx, \quad \approx_{m}
$$

are defined in the next two subsections.

3.2. The reduced category $\operatorname{red}(\mathcal{K})$. Let $\mathfrak{g}_{1}, \mathfrak{g}_{2} \in \operatorname{Mor}(\beta, \alpha)$. We say that they are $\approx$-equivalent if for any unitary representation of $\mathbb{G}$ we have $\widetilde{\rho}_{\alpha \beta}\left(\mathfrak{g}_{1}\right)=\widetilde{\rho}_{\alpha \beta}\left(\mathfrak{g}_{2}\right)$. The reduced category $\operatorname{red}(\mathcal{K})$ is the category whose objects are nonnegative integers and morphisms $\beta \rightarrow \alpha$ are $\approx$-equivalence classes of $\operatorname{Mor}(\beta, \alpha)$. Denote by $\operatorname{red}(\Gamma(m))$ semigroups of endomorphisms of $\operatorname{red}(\mathcal{K})$.

Also we define a weaker equivalence, $\mathfrak{g}_{1} \approx_{m} \mathfrak{g}_{2}$ if $\widetilde{\rho}_{\alpha \beta}\left(\mathfrak{g}_{1}\right)=\widetilde{\rho}_{\alpha \beta}\left(\mathfrak{g}_{1}\right)$ for all $\rho$ of height $\geqslant m$. Denote by $\operatorname{red}_{m}(\mathcal{K})$ the corresponding $m$-reduced category.

Our proof of Theorem 1.5 is based on an examination of the categories $\operatorname{red}(\mathcal{K})$ and $\operatorname{red}_{m}(\mathcal{K})$. We obtain an information sufficient for a classification of representations of $\mathbb{G}$. However, the author does not know an answer to Question 3.1 .

Question 3.1. Find a transparent description of the category $\operatorname{red}(\mathcal{K})$.

3.3. Inverse semigroups. Let $\mathcal{P}$ be a finite semigroup with an involution $x \mapsto x^{*}$. Then the following conditions are equivalent.

(A) $\mathcal{P}$ admits a faithful representation in a Hilbert space.

(B) $\mathcal{P}$ admits an embedding to a semigroup of partial bijection 7 of a finite set compatible with the involutions in $\mathcal{P}$ and in partial bijections.

(C) $\mathcal{P}$ is an inverse semigroup (see [6], 17], [16]), i.e., for any $x$ we have

$$
x x^{*} x=x, \quad x^{*} x x^{*}=x^{*}
$$

and any two idempotents in $\mathcal{P}$ commute.

Discuss briefly some properties of inverse semigroups. Any idempotent in $\mathcal{P}$ is self-adjoint, and for any $x$, the element $x^{*} x$ is an idempotent. Since idempotents commute, a product of idempotents is an idempotent. The semigroup of idempotents has a natural partial order,

$$
x \preceq y \quad \text { if } \quad x y=x .
$$

We have $x y \preceq x$. If $x \preceq y$ and $u \preceq v$, then $x u \preceq y v$. Since our semigroup is finite, the product of all idempotents is a minimal idempotent $\mathbf{0}$; we have $\mathbf{0} x=x \mathbf{0}=\mathbf{0}$ for any $x$.

Let $\mathcal{R}$ be a finite semigroup with involution. Then there exists an inverse semi$\operatorname{group} \operatorname{inv}(\mathcal{R})$ and epimorphism $\pi: \mathcal{R} \rightarrow \operatorname{inv}(\mathcal{R})$ such that any homomorphism $\psi$ from $\mathcal{R}$ to an inverse semigroup $\mathcal{Q}$ has the form $\psi=\varkappa \pi$ for some homomorphism $\varkappa: \operatorname{inv}(\mathcal{R}) \rightarrow \mathcal{Q}$. We say that $\operatorname{inv}(\mathcal{R})$ is the maximal inverse semigroup quotient of $\mathcal{R}$.

Lemma 3.1. The semigroups $\Gamma(m)$ are finite.

This is a corollary of the following statement; see [27, Lemma 4.1.a].

Lemma 3.2. Any double coset in $\mathbb{G}(m) \backslash \mathbb{G} / \mathbb{G}(m)$ has a representative in $\mathrm{GL}(3 m)$.

\footnotetext{
${ }^{7}$ Recall that a partial bijection $\sigma$ from a set $A$ to a set $B$ is a bijection from a subset $S$ of $A$ to a subset $T$ of $B$; see e.g., [17] or [20, Sect. VIII.1]. The adjoint partial bijection $\sigma^{*}: B \rightarrow A$ is the inverse bijection $T$ to $S$.
} 
We consider the following quotients of $\Gamma(m)$ :

(1) $\operatorname{inv}(\Gamma(m))$ is the maximal inverse semigroup quotient of $\Gamma(m)$;

(2) $\operatorname{red}(\Gamma(m)):=\operatorname{End}_{\text {red } \mathcal{K}}(m)$;

(3) $\operatorname{red}_{m}(\Gamma(m)):=\operatorname{End}_{\operatorname{red}_{m}(\mathcal{K})}(m)$.

We have the following sequence of epimorphism: 8 :

$$
\Gamma(m) \rightarrow \operatorname{inv}(\Gamma(m)) \rightarrow \operatorname{red}(\Gamma(m)) \rightarrow \operatorname{red}_{m}(\Gamma(m)) .
$$

For $g \in \mathbb{G}_{\text {fin }}$ we denote by $[g]_{m m}$ the corresponding element of $\Gamma(m)$ and by $[[g]]_{m m}$ the corresponding element of $\operatorname{inv}(\Gamma(m))$. The equality in $\Gamma(m)$ we denote by $\sim$, in inv $\Gamma((m))$ by $\equiv$, in $\operatorname{red}(\Gamma(m))$ by $\approx$, in $\operatorname{red}_{m}(\Gamma(m))$ by $\approx_{m}$. Denote by $\left[\left[g_{1}\right]\right] \diamond\left[\left[g_{2}\right]\right]$ the product in $\operatorname{inv}(\Gamma(m))$.

Our next purpose is to present some (non-maximal) inverse semigroup quotients of $\Gamma(m)$.

3.4. The category $\mathcal{L}$ of partial isomorphisms. Let $V, W$ be modules over $\mathbb{Z}_{p^{\mu}}$. A partial isomorphism $p: V \rightarrow W$ is an isomorphism of a submodule $A \subset V$ to a submodule $B \subset W$. We denote $\operatorname{dom} p:=A, \operatorname{im} p:=B$. By $p^{*}$ we denote the inverse map $B \rightarrow A$. Let $p: V \rightarrow W, q: W \rightarrow Y$ be partial isomorphisms. Then the product $p q$ is defined in the following way:

$$
\operatorname{dom} p q:=p^{*}(\operatorname{dom} q) \cap \operatorname{dom} p,
$$

for $v \in \operatorname{dom} p q$ we define $v(p q)=(v p) q$.

A partial isomorphism $p$ is an idempotent if $\operatorname{dom} p=\operatorname{im} p$ and $p$ is an identical map.

Objects of the category $\mathcal{L}$ are modules

$$
\mathfrak{l}_{+}^{\alpha} \oplus \mathfrak{l}_{-}^{\alpha}:=\left(\mathbb{Z}_{p^{\mu}}\right)^{\alpha} \oplus\left(\mathbb{Z}_{p^{\mu}}\right)^{\alpha}
$$

equipped with the following pairing

$$
\left\{v_{+} ; v_{-}\right\}:=\sum_{j} v_{+}^{j} v_{-}^{j}=v_{+}\left(v_{-}\right)^{t},
$$

where $v_{ \pm} \in \mathfrak{l}_{ \pm}^{\alpha}$. We say that two partial isomorphisms

$$
\xi_{+}: \mathfrak{l}_{+}^{\alpha} \rightarrow \mathfrak{l}_{+}^{\beta}, \quad \xi_{-}: \mathfrak{l}_{-}^{\alpha} \rightarrow \mathfrak{l}_{-}^{\beta}
$$

are compatible if for any $y_{+} \in \operatorname{dom} \xi_{+}$and $y_{-} \in \operatorname{dom} \xi_{-}$, we have

$$
\left\{\xi_{+}\left(y_{+}\right), \xi_{-}\left(y_{-}\right)\right\}=\left\{y_{+}, y_{-}\right\} .
$$

Next, we define a category $\mathcal{L}$. Its objects are spaces $\mathfrak{l}_{+}^{\alpha} \oplus \mathfrak{l}_{-}^{\alpha}$ and morphisms are pairs of compatible partial isomorphisms $\xi_{+}: \mathfrak{l}_{+}^{\alpha} \rightarrow \mathfrak{l}_{+}^{\beta}, \xi_{-}: \mathfrak{l}_{-}^{\alpha} \rightarrow \mathfrak{l}_{-}^{\beta}$.

The category $\mathcal{L}$ is equipped with an involution

$$
\left(\xi_{+}, \xi_{-}\right)^{*}=\left(\xi_{+}^{*}, \xi_{-}^{*}\right)
$$

and an automorphism

$$
\left(\xi_{+}, \xi_{-}\right)^{\star}=\left(\xi_{-}, \xi_{+}\right)
$$

Lemma 3.3. The semigroups $\operatorname{End}_{\mathcal{L}}(m)$ are inverse.

Indeed, $\operatorname{End}_{\mathcal{L}}(m)$ is a semigroup of partial bijections of a finite set $\mathfrak{l}_{+}^{m} \oplus \mathfrak{l}_{-}^{m}$. The whole category $\mathcal{L}$ is inverse for the same reason.

\footnotetext{
${ }^{8}$ All these semigroups are different.
} 
3.5. The functor $\Pi: \mathcal{K} \rightarrow \mathcal{L}$. Consider $g \in \mathbb{G}_{\text {fin }}$. Let actually $g$ be contained in $\mathrm{GL}(N)$. Represent $g$ as a block $(\beta+(N-\beta)) \times(\alpha+(N-\alpha))$ matrix and $g^{-1}$ as an $(\alpha+(N-\alpha)) \times(\beta+(N-\beta))$-matrix,

$$
g=\left(\begin{array}{cc}
a & b \\
c & d_{\star}
\end{array}\right), \quad g^{-1}=\left(\begin{array}{cc}
A & B \\
C & D_{\star}
\end{array}\right) .
$$

Define maps $\xi_{ \pm}: \mathfrak{l}_{ \pm}^{\alpha} \rightarrow \mathfrak{l}_{ \pm}^{\beta}$ by:

- $\operatorname{dom} \xi_{+}:=\operatorname{ker} b$ and $\xi_{+}$is the restriction of $a$ to $\operatorname{ker} b$;

- $\operatorname{dom} \xi_{-}:=\operatorname{ker} C^{t}$ and $\xi_{-}$is the restriction of $A^{t}$ to $\operatorname{ker} C^{t}$.

\section{Proposition 3.4.}

(a) The pair $\xi_{+}, \xi_{-}$depends only on the double coset containing $\mathfrak{g}$.

(b) Partial isomorphisms $\xi_{+}$, $\xi_{-}$are compatible.

(c) The map $\mathfrak{g} \mapsto\left(\xi_{+}, \xi_{-}\right)$determines a functor from the category $\mathcal{K}$ to the category $\mathcal{L}$.

Denote this functor by $\Pi$. Вy $\Pi(\mathfrak{g})$ we denote the morphism of $\mathcal{L}$ corresponding to $\mathfrak{g}$. We have

$$
\Pi\left(\mathfrak{g}^{*}\right)=(\Pi(\mathfrak{g}))^{*}, \quad \Pi\left(\mathfrak{g}^{\star}\right)=(\Pi(\mathfrak{g}))^{\star} .
$$

Proof. For any invertible matrix $v$ we have, $\operatorname{ker} b=\operatorname{ker} b v$. Therefore $\xi_{+}$depends only on a double coset. For $\xi_{-}$we apply (3.2).

(b) Let $v \in \operatorname{ker} b, w \in \operatorname{ker} C^{t}$. Then

$$
\{v, w\}=v w^{t}=v(a A+b C) w^{t}=v a \cdot\left(w A^{t}\right)^{t}+v b \cdot\left(w C^{t}\right)^{t}=\left\{v a, w A^{t}\right\}+0 .
$$

(c) We look to formula (2.4) for a product in $\mathcal{K}$. The new $\xi_{+}$is a restriction of $a p$ to $\operatorname{ker} b \cap \operatorname{ker} a q$. This is the product of two $\xi$-es.

Remark. According Ol'shanskǐ [30], for the case $\mathrm{GL}\left(\infty, \mathbb{F}_{p}\right)$ the functor $\Pi: \mathcal{K} \rightarrow \mathcal{L}$ determines an isomorphism of categories $\operatorname{red}(\mathcal{K}) \rightarrow \mathcal{L}$. However, for $\mu>1$ the maps $\Pi: \operatorname{red}(\Gamma(m)) \rightarrow \operatorname{Mor}_{\mathcal{L}}(m)$ are neither surjective nor injective. However we will observe that $\Pi$ induces isomorphisms of semigroups of idempotents; this provides us an important argument for the proof of Proposition 6.1

\section{Idempotents IN $\operatorname{inv}(\Gamma(m))$}

Here we examine idempotents in the semigroup $\operatorname{inv}(\Gamma(m))$. The main statement of the section is Proposition 4.10.

4.1. Projectors 9: $P_{\alpha}$. Consider an irreducible representation $\rho$ of $\mathbb{G}$; let subspaces $H_{m} \subset H$ and orthogonal projectors $P_{m}: H \rightarrow H_{m}$ be as above.

\section{Lemma 4.1.}

(a) The projector

$$
\left.P_{\alpha}\right|_{H_{m}}: H_{m} \rightarrow H_{\alpha}
$$

\footnotetext{
${ }^{9}$ This subsection contains generalities; $\mathcal{K}$ is an ordered category in the sense of [20 Sect. III.4]; this implies all statements of the subsection.
} 
is given by the operator $\widetilde{\rho}_{m m}\left(\Theta_{[m]}^{\alpha}\right)$, where

$$
\Theta_{[m]}^{\alpha}:=\left[\begin{array}{cc|cc}
1_{\alpha} & 0 & 0 & 0 \\
0 & 0 & 1_{m-\alpha} & 0 \\
\hline 0 & 1_{m-\alpha} & 0 & 0 \\
0 & 0 & 0 & 1_{\infty}
\end{array}\right]_{m m} \in \Gamma(m) .
$$

(b) The tautological embedding $H_{\alpha} \rightarrow H_{m}$ is defined by the operator $\widetilde{\rho}_{m \alpha}\left(\Lambda_{[m]}^{\alpha}\right)$, where

$$
\Lambda_{[m]}^{\alpha}:=\left[\begin{array}{cc|c}
1_{\alpha} & 0 & 0 \\
\hline 0 & 1_{m \alpha} & 0 \\
0 & 0 & 1_{\infty}
\end{array}\right]_{\alpha m} \in \operatorname{Mor}_{\mathcal{K}}(\alpha, m) .
$$

(c) The orthogonal projector $H_{m} \rightarrow H_{\alpha}$ is given by $\widetilde{\rho}_{\alpha m}\left(\left(\Lambda_{[m]}^{\alpha}\right)^{*}\right)$

$$
\left(\Lambda_{m}^{\alpha}\right)^{*}:=\left[\begin{array}{c|cc}
1_{\alpha} & 0 & 0 \\
0 & 1_{m-\alpha} & 0 \\
\hline 0 & 0 & 1_{\infty}
\end{array}\right]_{m \alpha} \in \operatorname{Mor}_{\mathcal{K}}(m, \alpha) .
$$

Proof. (a) We apply Proposition 2.1(a). For $j>m-\alpha$ we have $\left[\theta^{\alpha}(j)\right]_{m m}=\Theta_{[m]}^{\alpha}$. The same argument proves (b) and (c).

\section{Lemma 4.2.}

(a) The map

$$
\iota_{m}^{\alpha}:\left[\begin{array}{c|c}
a & b \\
\hline c & d_{\star}
\end{array}\right]_{\alpha \alpha} \mapsto\left[\begin{array}{cc|cc}
a & 0 & b & 0 \\
0 & 0 & 0 & 1_{m-\alpha} \\
\hline c & 0 & d & 0 \\
0 & 1_{m-\alpha} & 0 & 0_{\star}
\end{array}\right]_{m m}
$$

is a homomorphism $\Gamma(\alpha) \rightarrow \Gamma(m)$.

(b) We have

$$
\iota_{m}^{\alpha}(\mathfrak{g}) \sim \Lambda_{n}^{\alpha} \circ \mathfrak{g} \circ\left(\Lambda_{n}^{\alpha}\right)^{*} .
$$

This follows from a straightforward calculation.

Corollary 4.3. The map $\iota_{m}^{\alpha}$ is compatible with representations $\widetilde{\rho}$ of $\Gamma(\alpha)$ and $\Gamma(m)$. Namely, operators $\widetilde{\rho}_{m m}\left(\iota_{m}^{\alpha}(\mathfrak{g})\right)$ have the following block structure with respect to the decomposition $H_{m}=H_{\alpha} \oplus\left(H_{m} \ominus H_{\alpha}\right)$ :

$$
\widetilde{\rho}_{m m}\left(\iota_{m}^{\alpha}(\mathfrak{g})\right)=\left(\begin{array}{cc}
\widetilde{\rho}_{\alpha \alpha}(\mathfrak{g}) & 0 \\
0 & 0
\end{array}\right) .
$$

4.2. Idempotents in $\operatorname{inv}(\Gamma(m))$. Here we formulate several lemmas (their proofs occupy Subsections 4.3 4.7); as a corollary we get Proposition 4.10.

Lemma 4.4. Let for

$$
[g]=\left[\begin{array}{cc}
a & b \\
c & d_{\star}
\end{array}\right]_{m m} \in \Gamma(m)
$$

one of the blocks $a, d$ be degenerate. Then $[[g]]_{m m} \in \operatorname{inv}(\Gamma(m))$ has a representative $\left[g^{\prime}\right]$, for which both blocks a, $d$ are degenerate.

Denote by

$$
\Gamma^{\circ}(m)
$$

the subsemigroup in $\Gamma(m)$ consisting of all $[g]$, for which both blocks $a, d$ are nondegenerate. 
Lemma 4.5. Any idempotent in $\operatorname{inv}(\Gamma(m))$ has a representative of the form $q$. $[[R]] \cdot q^{-1}$ with $q$ ranging $\mathrm{GL}(m)$ and $R$ having the form

$$
[R]:=\left[\begin{array}{cc|cc}
1_{\alpha} & 0 & \varphi & 0 \\
0 & 0 & 0 & 1_{m-\alpha} \\
\hline \psi & 0 & \varkappa & 0 \\
0 & 1_{m-\alpha} & 0 & 0_{\star}
\end{array}\right]_{m m} \in \Gamma(m),
$$

where

$$
\left[\begin{array}{c|c}
1 & \varphi \\
\hline \psi & \varkappa_{\star}
\end{array}\right]_{\alpha \alpha} \in \Gamma(\alpha)
$$

represents an idempotent in $\operatorname{inv}\left(\Gamma^{\circ}(\alpha)\right)$. The parameter $\alpha$ ranges in the set $0,1,2$, $\ldots, m$.

Remark. Denote

$$
R^{\square}:=\left[\begin{array}{cc|c}
1_{\alpha} & 0 & \varphi \\
0 & 1_{m-\alpha} & 0 \\
\hline \psi & 0 & \varkappa_{\star}
\end{array}\right]_{m m} .
$$

Then the following elements of $\Gamma(m)$ coincide:

$$
R=R^{\square} \Theta_{m}^{\alpha}=\Theta_{m}^{\alpha} R^{\square}=\Theta_{m}^{\alpha} R^{\square} \Theta_{m}^{\alpha} .
$$

Denote

$$
X(b, c):=\left(\begin{array}{c|cc}
1_{m} & b & 0 \\
\hline 0 & 1 & 0 \\
c & 0 & 1_{\star}
\end{array}\right) \in \mathbb{G}_{\mathrm{fin}} .
$$

Lemma 4.6. Elements of the form $[X(b, c)]$ are idempotents in $\Gamma^{\circ}(m)$. They depend only on $\operatorname{ker} b$ and $\operatorname{ker} c^{t} \subset \mathfrak{l}^{m}$.

Let $L:=\operatorname{ker} b$ and $M:=\operatorname{ker} c^{t}$. Denote

$$
X[L, M]:=[X(b, c)] .
$$

Lemma 4.7. We have

$$
\mathcal{X}\left[L_{1}, M_{1}\right] \mathcal{X}\left[L_{2}, M_{2}\right]=X\left[L_{1} \cap L_{2}, M_{1} \cap M_{2}\right] .
$$

Lemma 4.8. Any idempotent in $\operatorname{inv}\left(\Gamma^{\circ}(m)\right)$ has the form $\mathcal{X}[L, M]$.

Corollary 4.9. Idempotents $\mathcal{X}[L, M]$ are pairwise distinct in $\operatorname{inv}\left(\Gamma^{\circ}(m)\right)$.

Proof. Indeed, $\operatorname{End}_{\mathcal{L}}(m)$ is an inverse semigroup; therefore we have a chain of maps

$$
\Gamma^{\circ}(m) \rightarrow \operatorname{inv}\left(\Gamma^{\circ}(m)\right) \rightarrow \operatorname{inv}(\Gamma(m)) \rightarrow \operatorname{Mor}_{\mathcal{L}}(m) .
$$

The image of $X(b, c)$ in $\operatorname{Mor}_{\mathcal{L}}(m)$ is precisely the pair of identical partial isomorphisms $M \rightarrow M, L \rightarrow L$. Therefore for nonequivalent $X(b, c)$ we have different images.

Proposition 4.10. Any idempotent in inv $(\Gamma(m))$ has a representative of the form

$$
q \cdot\left[\begin{array}{cc|ccc}
1_{\alpha} & 0 & b & 0 & 0 \\
0 & 0 & 0 & 0 & 1_{m-\alpha} \\
\hline 0 & 0 & 1 & 0 & 0 \\
c & 0 & 0 & 1 & 0 \\
0 & 1_{m-\alpha} & 0 & 0 & 0
\end{array}\right]_{m m} \cdot q^{-1},
$$

where $q \in \mathrm{GL}(m)=\operatorname{Aut}_{\mathcal{K}}(m)$. 
Proof. Lemma 4.2 defines a canonical embedding $i_{m}^{\alpha}: \Gamma(\beta) \rightarrow \Gamma(m)$ for $\alpha<m$. By Lemma 4.5 any idempotent in $\operatorname{inv}(\Gamma(m))$ is equivalent to an idempotent lying in some $i_{m}^{\alpha}\left(\Gamma^{\circ}(\alpha)\right)$. Lemma 4.8 gives us a canonical form of this idempotent.

Now we start proofs of Lemmas 4.44 .8

4.3. Proof of Lemma 4.4. Clearly $\Gamma(m) \backslash \Gamma^{\circ}(m)$ is a two-sided ideal in $\Gamma(m)$. Since $\left[\left[g \circ\left(g^{-1} \circ g\right)\right]\right]_{m m}=[[g]]_{m m}$, it is sufficient to prove the statement for idempotents.

Let

$$
g=\left(\begin{array}{cc}
a & b \\
c & d_{\star}
\end{array}\right), \quad g^{-1}=:\left(\begin{array}{cc}
A & B \\
C & D_{\star}
\end{array}\right) .
$$

Then

$$
[[g]] \diamond[[g]]^{*} \equiv\left[\left[g \circ g^{-1}\right]\right] \equiv\left[\left[\begin{array}{c|c}
a A & * \\
\hline * & *
\end{array}\right]\right] .
$$

If $a$ is degenerate, then $a A$ is degenerate. Now let $a$ be non-degenerate, $d$ degenerate. Since the matrices (4.5) are inverse one to another, we have

$$
a A=1-b C, \quad D d=1-C b .
$$

We see that $(1-C b)(\bmod p)$ is degenerate, $(1-b C)(\bmod p)$ also is degenerate, and therefore $a A$ is degenerate.

\subsection{Proof of Lemma 4.5.}

Step 1.

Lemma 4.11. Let $x$ be an idempotent in $\operatorname{inv}(\Gamma(m))$. Then it can be represented as $[[u]]$, where $u=u^{-1}$.

Proof. Let $x=[[g]]$. Then

$$
x=[[g]] \diamond[[g]]^{*}=\left[\left[g \circ g^{-1}\right]\right]=\left[\left[g \theta^{m}(j) g^{-1}\right]\right]
$$

for sufficiently large $j$. We set $u:=g \theta^{m}(j) g^{-1}$.

Lemma 4.12. Let $g=g^{-1} \in \mathbb{G}_{\mathrm{fin}}$. For any $N>0$ there exists a representative $r \in \mathbb{G}_{\text {fin }}$ of $[g]^{\circ 2 N}$ such that $r=r^{-1}$.

Proof. Let actually $g \in \mathrm{GL}(m+l)$. Then we choose the following representative of $[g]^{\circ 8}$ :

$$
r=g \theta^{m}(l) g \theta^{m}(2 l) g \theta^{m}(4 l) g \theta^{m}(8 l) g \theta^{m}(4 l) g \theta^{m}(2 l) g \theta^{m}(l) g .
$$

Step 2.

Lemma 4.13. Let $g=g^{-1}=\left(\begin{array}{cc}a & b \\ c & d_{\star}\end{array}\right) \in \mathbb{G}$. Then there exists a matrix

$$
Z=\left(\begin{array}{c|c}
\zeta & 0 \\
\hline 0 & 1_{\star}
\end{array}\right) \in \operatorname{Aut}_{\mathcal{K}}(m), \quad \text { where } \zeta \in \operatorname{GL}(m),
$$

and $N$ such that

$$
\left[\left[Z \cdot g \cdot Z^{-1}\right]\right]^{\diamond N}=\left[\left[\left(\begin{array}{cc}
\zeta & 0 \\
0 & 1_{\star}
\end{array}\right)\left(\begin{array}{cc}
a & b \\
c & d_{\star}
\end{array}\right)\left(\begin{array}{cc}
\zeta & 0 \\
0 & 1_{\star}
\end{array}\right)^{-1}\right]\right]^{\diamond N}
$$


has a form

$$
r=\left[\left[\begin{array}{cc|c}
0 & 0 & * \\
0 & 1_{k} & * \\
\hline * & * & *
\end{array}\right]\right]
$$

where $k$ is the rank of the reduced matrix $a^{m}(\bmod p)$.

Clearly our lemma is a corollary of the following statement:

Lemma 4.14. For any $m \times m$ matrix a over $\mathbb{Z}_{p^{\mu}}$ there exists $\zeta \in \mathrm{GL}(m)$ and $N$ such that

$$
\left(\zeta a \zeta^{-1}\right)^{N}=\left(\begin{array}{cc}
0 & 0 \\
0 & 1_{k}
\end{array}\right) .
$$

Proof. We split the operator $a(\bmod p)$ over the field $\mathbb{F}_{p}$ as a direct sum of a nilpotent part $S$ and an invertible part $T$. For sufficiently large $M$ the matrix $\left(\begin{array}{cc}S & 0 \\ 0 & T\end{array}\right)^{M}$ has the form $\left(\begin{array}{ll}0 & 0 \\ 0 & P\end{array}\right)$ with a nondegenerate $P$. Since the group $\operatorname{GL}\left(k, \mathbb{F}_{p}\right)$ is finite, $P^{L}=1_{k}$ for some $L$.

Thus without a loss of generality, we can assume that $a$ has a form

$$
a=\left(\begin{array}{cc}
p \alpha & p \beta \\
p \gamma & 1+p \delta
\end{array}\right)
$$

where $\alpha, \beta, \gamma, \delta$ are matrices over $\mathbb{Z}_{p^{\mu}}$. We conjugate it as follows

$$
\left(\begin{array}{cc}
1 & p u \\
0 & 1
\end{array}\right)\left(\begin{array}{cc}
p \alpha & p \beta \\
p \gamma & 1+p \delta
\end{array}\right)\left(\begin{array}{cc}
1 & -p u \\
0 & 1
\end{array}\right)=\left(\begin{array}{ll}
* & -p^{2}(\alpha u+u \gamma u)+p(\beta+u(1+p \delta)) \\
* & *
\end{array}\right) .
$$

We wish to choose $u$ to make zero in the boxed block. It is sufficient to find a matrix $u$ satisfying the following equation:

$$
u=(-\beta+p(\alpha u+u \gamma u))(1+p \delta)^{-1}=-\beta+p(-\delta+\alpha u+u \gamma u)(1+p \delta)^{-1} .
$$

We look for a solution in the form

$$
u=\sum_{k=0}^{\mu} p^{k} S_{k}
$$

First, we consider $S_{k}$ as formal noncommutative variables. Then we get a system of equations of the form

$$
S_{0}=-\beta, \quad S_{k}=F_{k}\left(\alpha, \beta, \gamma, \delta ; S_{0}, S_{1}, \ldots, S_{k-1}\right),
$$

where $F_{k}$ are polynomial expressions with integer coefficients. These equations can be regarded as recurrence formulas for $S_{k}$. In this way we get a solution $u$.

Thus without a loss of generality we can assume that $a$ has the form

$$
a=\left(\begin{array}{cc}
p \alpha^{\prime} & 0 \\
p \gamma^{\prime} & 1+p \delta^{\prime}
\end{array}\right)
$$

Raising it to $\mu$-th power, we come to a matrix of the form

$$
a=\left(\begin{array}{cc}
0 & 0 \\
p \gamma^{\prime \prime} & 1+p \delta^{\prime \prime}
\end{array}\right) .
$$


We conjugate it as

$$
\left(\begin{array}{cc}
1 & 0 \\
p v & 1
\end{array}\right)\left(\begin{array}{cc}
0 & 0 \\
p \gamma^{\prime \prime} & 1+p \delta^{\prime \prime}
\end{array}\right)\left(\begin{array}{cc}
1 & 0 \\
-p v & 1
\end{array}\right)=\left(\begin{array}{cc}
0 & 0 \\
p\left(\gamma^{\prime \prime}-\left(1+p \delta^{\prime \prime}\right) v\right) & \left(1+\delta^{\prime \prime} v\right)
\end{array}\right) .
$$

Taking $v=\left(1+p \delta^{\prime \prime}\right)^{-1} \gamma^{\prime \prime}$ we kill the left lower block and come to a matrix of the form $\left(\begin{array}{cc}0 & 0 \\ 0 & 1+p \delta^{\prime \prime \prime}\end{array}\right)$. Raising it in $p^{\mu-1}$-th power we come to $\left(\begin{array}{ll}0 & 0 \\ 0 & 1\end{array}\right)$.

Step 3. Thus the element $[[g]]^{\diamond 2 N}$ from Lemma 4.13 has a representative of the following block $(m-k)+k+(m-k)+\infty$ form:

$$
r=r^{-1}=\left(\begin{array}{cc|cc}
0 & 0 & \beta_{11} & \beta_{12} \\
0 & 1_{k} & \beta_{21} & \beta_{22} \\
\hline \gamma_{11} & \gamma_{12} & \delta_{11} & \delta_{12} \\
\gamma_{21} & \gamma_{22} & \delta_{21} & \delta_{22 \star}
\end{array}\right) .
$$

Lemma 4.15. There is a matrix $U=\left(\begin{array}{c|c}1_{m} & 0 \\ \hline 0 & u_{\star}\end{array}\right)$ such that $U r U^{-1}$ has the form

$$
\widetilde{r}=\left(\begin{array}{cc|cc}
0 & 0 & 1_{m-k} & 0 \\
0 & 1_{k} & 0 & \varphi \\
\hline 1_{m-k} & 0 & 0 & 0 \\
0 & \psi & 0 & \varkappa_{\star}
\end{array}\right) .
$$

Recall that $[r] \sim\left[U r U^{-1}\right]$.

Proof. Since the matrix $\left(\beta_{11} \quad \beta_{12}\right)$ is nondegenerate (otherwise $r$ is degenerate), we can choose a conjugation of $r$ by matrices $U=\left(\begin{array}{cc}1_{m} & 0 \\ 0 & *\end{array}\right)$ reducing this block to the form $\left(\begin{array}{ll}1 & 0\end{array}\right)$. We have $r^{2}=1$; evaluating $r^{2}$ we get $\gamma_{11}$ in the left upper block. Therefore $\gamma_{11}=1$. Thus we come to new $r$,

$$
r^{\sim}=\left(\begin{array}{cc|cc}
0 & 0 & 1 & 0 \\
0 & 1_{k} & \beta_{21} & \beta_{22} \\
\hline 1 & \gamma_{12} & \delta_{11} & \delta_{12} \\
\gamma_{21} & \gamma_{22} & \delta_{21} & \delta_{22 \star}
\end{array}\right)
$$

with new $\beta, \gamma, \delta$. Next, we conjugate this matrix by

$$
\left(\begin{array}{c|cc}
1_{m} & 0 & 0 \\
\hline 0 & 1_{m-k} & 0 \\
0 & -\gamma_{21} & 1_{\star}
\end{array}\right)
$$

and kill $\gamma_{21}$. Thus we come to new $r$,

$$
r^{\sim \sim}=\left(\begin{array}{cc|cc}
0 & 0 & 1 & 0 \\
0 & 1_{k} & \beta_{21} & \beta_{22} \\
\hline 1 & \gamma_{12} & \delta_{11} & \delta_{12} \\
0 & \gamma_{22} & \delta_{21} & \delta_{22 \star}
\end{array}\right) .
$$

But $\left(r^{\sim \sim}\right)^{2}=1$. Looking to third row and third column of $\left(r^{\sim \sim}\right)^{2}$ we observe that

$$
\beta_{21}, \delta_{11}, \delta_{21}, \gamma_{12}, \delta_{12} \text { are zero. }
$$

Thus, $r^{\sim \sim}$ has the desired form. 


\subsection{Proof of Lemma 4.6. Denote}

$$
\left[X_{+}(A)\right]:=\left[\begin{array}{cc}
1 & A \\
0 & 1_{\star}
\end{array}\right] .
$$

We can conjugate this matrix by $\left(\begin{array}{cc}1 & 0 \\ 0 & u_{\star}\end{array}\right)$. Therefore a matrix $A$ is defined up to multiplications $A \sim A u$, where $u$ is an invertible matrix. The invariant of this action is $\operatorname{ker} A$ (this is more or less clear; formally we can refer to Lemma 7.3 proved below).

Next,

$$
\left[X_{+}(A)\right] \circ\left[X_{+}(A)\right]=\left[\begin{array}{c|cc}
1 & A & A \\
\hline 0 & 1 & 0 \\
0 & 0 & 1_{\star}
\end{array}\right] .
$$

We have $\operatorname{ker}\left(\begin{array}{ll}A & A\end{array}\right)=\operatorname{ker} A$ and therefore $\left[X_{+}(A)\right]$ is an idempotent. In the same way, $\left[X_{-}(B)\right]:=\left[\begin{array}{cc}1 & 0 \\ B & 1_{\star}\end{array}\right]$ is an idempotent. It remains to notice that

$$
[X(A, B)]=\left[X_{+}(A)\right] \diamond\left[X_{-}(B)\right] .
$$

Thus $[X(A, B)]$ is an idempotent.

4.6. Proof of Lemma 4.6. In notation of the previous subsection

$$
X_{-}\left(A_{1}\right) \circ X_{-}\left(A_{2}\right) \sim X\left(\left(\begin{array}{ll}
A_{1} & A_{2}
\end{array}\right)\right),
$$

i.e.,

$$
X\left[\operatorname{ker} A_{1}, 0\right] \diamond X\left[\operatorname{ker} A_{2}, 0\right] \equiv X\left[\operatorname{ker} A_{1} \cap \operatorname{ker} A_{2}, 0\right],
$$

or

$$
X\left[L_{1}, 0\right] \diamond X\left[L_{2}, 0\right] \equiv X\left[L_{1} \cap L_{2}, 0\right] .
$$

On the other hand, we have

$$
\mathcal{X}[L, 0] \diamond \mathcal{X}[0, M] \equiv X[L, M],
$$

and now the statement becomes obvious.

Proof of Lemma 4.7. Indeed,

$$
\left[X\left(b_{1}, c_{1}\right)\right] \circ\left[X\left(b_{1}, c_{1}\right)\right] \sim\left[X\left(\left(\begin{array}{ll}
b_{1} & b_{2}
\end{array}\right),\left(\begin{array}{l}
c_{1} \\
c_{2}
\end{array}\right)\right)\right]
$$

and $\operatorname{ker}\left(b_{1} \quad b_{2}\right)=\operatorname{ker} b_{1} \cap \operatorname{ker} b_{2}$.

\subsection{Proof of Lemma 4.8.}

Step 1. Any idempotent $[[g]] \in \operatorname{inv}\left(\Gamma^{\circ}(m)\right)$ has a representative of the form $\left(\begin{array}{cc}1 & a \\ b & 1_{\star}\end{array}\right)$, where $a b=0$.

Let $[[g]]=\left[\left[\begin{array}{cc}\alpha & \beta \\ \gamma & \delta_{\star}\end{array}\right]\right]$ be an idempotent; let $\alpha, \delta$ be nondegenerate. By Lemma 4.11without loss of generality we can assume $g=g^{-1}$. Taking an appropriate power $[r]=[g]^{\circ 2 N}$, we can achieve $\alpha=1$. By Lemma 4.12, we can assume $r=r^{-1}$.

Set $r=\left(\begin{array}{cc}1 & -a \\ b & c_{\star}\end{array}\right)$. Evaluating $r^{2}=1$ we get the following collection of conditions

$$
a b=0, \quad a c=-a, \quad c b=-b, \quad c^{2}-b a=1 .
$$


We replace $r$ by an equivalent matrix

$$
r \sim\left(\begin{array}{cc}
1 & -a \\
b & c_{\star}
\end{array}\right)\left(\begin{array}{cc}
1 & 0 \\
0 & c_{\star}^{-1}
\end{array}\right)=\left(\begin{array}{cc}
1 & -a c^{-1} \\
b & 1_{\star}
\end{array}\right)=\left(\begin{array}{cc}
1 & a \\
b & 1_{\star}
\end{array}\right),
$$

here we used the identity $-a c^{-1}=a$.

Step 2. We evaluate $[r]^{\circ 2}$,

$$
\begin{gathered}
{[r]^{\circ 2}=\left[\left(\begin{array}{c|cc}
1 & a & 0 \\
\hline b & 1 & 0 \\
0 & 0 & 1_{\star}
\end{array}\right)\left(\begin{array}{c|cc}
1 & 0 & a \\
\hline 0 & 1 & 0 \\
b & 0 & 1_{\star}
\end{array}\right)\right]=\left[\begin{array}{c|cc}
1 & a & a \\
\hline b & 1 & b a \\
b & 0 & 1_{\star}
\end{array}\right]} \\
\\
\left.\sim\left[\begin{array}{c|cc}
1 & a & a \\
\hline b & 1 & b a \\
b & 0 & 1_{\star}
\end{array}\right)\left(\begin{array}{c|cc}
1 & 0 & 0 \\
\hline 0 & 1 & -b a \\
0 & 0 & 1_{\star}
\end{array}\right)\right]=\left[\begin{array}{c|cc}
1 & a & a-a b a \\
\hline b & 1 & 0 \\
b & 0 & 1_{\star}
\end{array}\right]
\end{gathered}
$$

But $a b=0$ and therefore $a b a=0$. Repeating the same reasoning, we get

$$
[[r]]^{\diamond N} \equiv[[q]] \equiv\left[\left[\begin{array}{c|ccc}
1_{m} & a & \ldots & a \\
\hline b & 1 & \ldots & 0 \\
\vdots & \vdots & \ddots & \vdots \\
b & 0 & \ldots & 1_{\star}
\end{array}\right]\right]
$$

Step 3. Next, we set $N=p^{\mu}$ in formula (4.8). Consider the following block matrix $u$ of size $p^{\mu}$,

$$
u:=\left(\begin{array}{ccccc}
1 & & & & \\
-1 & 1 & & & \\
0 & -1 & 1 & & \\
\vdots & \vdots & \vdots & \ddots & \\
0 & 0 & 0 & \ldots & 1
\end{array}\right), \quad u^{-1}:=\left(\begin{array}{ccccc}
1 & & & & \\
1 & 1 & & & \\
1 & 1 & 1 & & \\
\vdots & \vdots & \vdots & \ddots & \\
1 & 1 & 1 & \ldots & 1
\end{array}\right) .
$$

We conjugate the matrix $q$ defined by (4.8) as

$$
\left(\begin{array}{cc}
1 & 0 \\
0 & u_{\star}
\end{array}\right) q\left(\begin{array}{cc}
1 & 0 \\
0 & u_{\star}^{-1}
\end{array}\right)
$$

We have

$$
u\left(\begin{array}{c}
b \\
b \\
b \\
\vdots
\end{array}\right)=\left(\begin{array}{c}
b \\
0 \\
0 \\
\vdots
\end{array}\right), \quad\left(\begin{array}{lllll}
a & a & a & a & \ldots
\end{array}\right) u^{-1}=\left(\begin{array}{lllll}
0 & -a & -2 a & -3 a & \ldots
\end{array}\right),
$$

and we get a matrix of the form $X(A, B)$.

\section{IDEMPotents In $\operatorname{red}(\Gamma(m))$}

Here the main statement is Proposition [5.1] which shows that all idempotents in $\operatorname{red}(\Gamma(m))$ have representatives in $\operatorname{red}\left(\Gamma^{\circ}(m)\right)$; therefore they have the form $X[L, M]$ ), where $L \subset \mathfrak{l}^{m}, M \subset \mathfrak{l}^{m}$. The second fact (Proposition [5.3), which is important for the proof below, is a coherence of elements $\mathcal{X}[L, M]$ in different semigroups $\operatorname{red}(\Gamma(n))$. 


\subsection{Coincidence of idempotents.}

Proposition 5.1. The following idempotents in $\operatorname{inv}(\Gamma(m))$ coincide as elements of $\operatorname{red}(\Gamma(m))$ :

$$
\begin{aligned}
{\left[\left[X_{\alpha}^{\bigcirc}(b, c)\right]\right]:=\left[\left[X \left(\left(\begin{array}{cc}
b & 0 \\
0 & 1_{m-\alpha}
\end{array}\right)\right.\right.\right.} & \left.\left.\left.,\left(\begin{array}{cc}
c & 0 \\
0 & 1_{m-\alpha}
\end{array}\right)\right)\right]\right] \\
= & {\left.\left[\begin{array}{cc|cccc}
1_{\alpha} & 0 & b & 0 & 0 & 0 \\
0 & 1_{m-\alpha} & 0 & 1_{m-\alpha} & 0 & 0 \\
\hline 0 & 0 & 1 & 0 & 0 & 0 \\
0 & 0 & 0 & 1 & 0 & 0 \\
c & 0 & 0 & 0 & 1 & 0 \\
0 & 1_{m-\alpha} & 0 & 0 & 0 & 1_{\star}
\end{array}\right]\right]_{m m}, }
\end{aligned}
$$

and

$$
\left[\left[X_{\alpha}^{\square}(b, c)\right]\right]:=\left[\left[\begin{array}{cc|ccc}
1_{\alpha} & 0 & b & 0 & 0 \\
0 & 0 & 0 & 0 & 1_{m-\alpha} \\
\hline 0 & 0 & 1 & 0 & 0 \\
c & 0 & 0 & 1 & 0 \\
0 & 1_{m-\alpha} & 0 & 0 & 0
\end{array}\right]\right]_{m m} .
$$

Corollary 5.2. Any idempotent in $\operatorname{red}(\Gamma(m))$ has the form $\mathcal{X}[L, M]$.

Proof of corollary. The semigroup $\operatorname{red} \Gamma(m))$ is a quotient of $\operatorname{inv}(\Gamma(m))$; the semigroup of idempotents also is a quotient of the semigroup of idempotents. By Proposition 4.10 all idempotents in $\operatorname{inv}(\Gamma(m))$ have $\left[\left[X_{\alpha}^{\bigcirc}[b, c]\right]\right.$. By Proposition [5.1, they also can be written as $\left[\left[X_{\alpha}^{\square}[b, c]\right]\right.$.

Proposition will be proved in Subsection 5.3.

Remarks.

(a) The idempotents $\left[\left[X_{\alpha}^{\bigcirc}(b, c)\right]\right]$ and $\left[\left[X_{\alpha}^{\square}(b, c)\right]\right]$ are different in $\operatorname{inv}(\Gamma(m))$. Indeed, we have the following homomorphism from $\Gamma(m)$ to the inverse semigroup $\operatorname{End}_{\mathcal{L}}(m)$. On $\Gamma^{\circ}(m)$ we define it as the map $\Pi$ described in Subsection 3.5. On the other hand, we send $\Gamma(m) \backslash \Gamma^{\circ}(m)$ to $\mathbf{0}$, i.e., to a pair of partial bijections with empty domains of definiteness. This map separates our idempotents.

(b) Idempotents $X[L, M]$ are pairwise different in $\operatorname{red}(\Gamma(m))$. To verify this, consider the representation of $\mathbb{G}$ in $\ell^{2}(\mathbb{G} / \mathbb{G}[L ; M])$. It is easy to show that $\chi(L, M)$ is the minimal idempotent of $\operatorname{red}(\Gamma(m))$ acting in this representation nontrivially.

5.2. Coherence. Let $L, M \subset \mathfrak{l}^{m}$ be submodules. Formula (4.4) defines the idempotent $X[L, M]=X(b, c)$ as an element of $\Gamma(m)$; recall that $L=\operatorname{ker} b, M=\operatorname{ker} c^{t}$. However, for $n>m$ we can regard $L, M \subset \mathfrak{l}^{m}$ as submodules $L$ in $\mathfrak{l}^{n} \supset \mathfrak{l}^{m}$. In the larger space we have

$$
L=\operatorname{ker}\left(\begin{array}{cc}
b & 0 \\
0 & 1_{n-m}
\end{array}\right), \quad M=\operatorname{ker}\left(\begin{array}{cc}
c & 0 \\
0 & 1_{n-m}
\end{array}\right) .
$$

Consider a unitary representation $\rho$ of $\mathbb{G}$ in a Hilbert space $H$. For any $n \geqslant m$ we have an operator

$$
\widetilde{\rho}_{n n}\left(X\left(\left(\begin{array}{cc}
b & 0 \\
0 & 1_{n-m}
\end{array}\right),\left(\begin{array}{cc}
c & 0 \\
0 & 1_{n-m}
\end{array}\right)\right)\right): H_{n} \rightarrow H_{n} .
$$

We claim that these operators as operators $H \rightarrow H$ depend only on $L, M$ and not on $n$. Precisely, we have the following statement. 


\section{Proposition 5.3.}

(a) Let $n \geqslant m$. Then a block matrix structure of the operator (5.1) with respect to the orthogonal decomposition $H_{n}=H_{m} \oplus\left(H_{n} \ominus H_{m}\right)$ is

$$
\widetilde{\rho}_{n n}\left(X\left(\left(\begin{array}{cc}
b & 0 \\
0 & 1_{n-m}
\end{array}\right),\left(\begin{array}{cc}
c & 0 \\
0 & 1_{n-m}
\end{array}\right)\right)\right)=\left(\begin{array}{cc}
\widetilde{\rho}_{m m}(X(b, c)) & 0 \\
0 & 0
\end{array}\right) .
$$

(b) For any $L, M \subset \mathfrak{l}^{m}$ we have a well-defined operator $\widetilde{\rho}(\mathcal{X}[L, M])$ in $H$, which sends $H_{m}$ to $H_{m}$ as $\widetilde{\rho}_{m m}(X[L, M])$ and is zero on the orthocomplement $H \ominus H_{m}$.

Proof. According Corollary 4.3, the right hand side of (5.2) is $\widetilde{\rho}_{n n}\left(X_{m}^{\square}(b, c)\right)$. By Proposition 5.1 this operator coincides with $\widetilde{\rho}_{n n}\left(X_{m}^{\bigcirc}(b, c)\right)$.

\subsection{Proof of Proposition 5.1.}

Lemma 5.4. Let $\mathfrak{g} \in \operatorname{red}(\Gamma(m))$ be an idempotent. Let $g$ be a representative of $\mathfrak{g}$ in $\mathbb{G}_{\mathrm{fin}}$. Then for any unitary representation $\rho$ of $\mathbb{G}$ in a Hilbert space $H$ the image of the orthogonal projector $\widetilde{\rho}_{m m}(\mathfrak{g})$ coincides with the space of fixed points of the subgroup in $\mathbb{G}$ generated by $\mathbb{G}(m)$ and $g$.

Proof. Let $v \in \operatorname{im} \widetilde{\rho}_{m m}(\mathfrak{g})$, i.e.,

$$
P_{m} \rho(g) P_{m} v=v .
$$

This happens if and only if $P_{m} v=v, \rho(g) v=v$. The condition $P_{m} v=v$ means that $\rho(h) v=v$ for all $h \in \mathbb{G}(m)$.

Therefore, it is sufficient to show that the group generated by $\mathbb{G}(m)$ and $X^{\bigcirc}(b, c)$ coincides with the group generated by $\mathbb{G}(m)$ and $X^{\square}(b, c)$.

Lemma 5.5. The group generated by the subgroup $\mathbb{G}(\beta)$ and the matrix

$$
X(1,1)=\left(\begin{array}{c|cc}
1_{\beta} & 1_{\beta} & 0 \\
\hline 0 & 1_{\beta} & 0 \\
1_{\beta} & 0 & 1_{\beta \star}
\end{array}\right)
$$

coincides with $\mathbb{G}$.

Proof. Denote by $G$ the group generated by $X(1,1)$ and $\mathbb{G}(\beta)$. Conjugating $X(1,1)$ by block diagonal matrices we can get any matrix of the form $X(A, B)$ with nondegenerate $A, B$. Multiplying such matrices we observe that elements of the form $X\left(A_{1}+A_{2}, B_{1}+B_{2}\right)$ are contained in $G$. In particular, $X(0,2) \in G$. Since $p \neq 2$, conjugating $X(0,2)$ by a block scalar matrix we come to $X(0,1) \in G$. In the same way $X(1,0) \in G$. Now the statement became more-or-less obvious.

Lemma 5.6. The group generated by $\mathbb{G}(\beta)$ and the matrix

$$
\left(\begin{array}{c|c}
0 & 1_{\beta} \\
\hline 1_{\beta} & 0_{\star}
\end{array}\right)
$$

coincides with $\mathbb{G}$.

Proof. Denote this group by $G$. Denote $S_{\infty}(\beta):=S_{\infty} \cap \mathbb{G}(\beta)$. Multiplying the matrix (5.3) from the left and right by elements of $S_{\infty}(\beta)$ we can get an arbitrary matrix of the form $\left(\begin{array}{c|c}0 & \sigma_{1} \\ \hline \sigma_{2} & 0_{\star}\end{array}\right)$ with $\sigma_{1}, \sigma_{2} \in S_{\beta}$. Multiplying two matrices of this type we can get any matrix $\left(\begin{array}{c|c}\sigma & 1 \\ \hline 0 & 1_{\star}\end{array}\right)$, where $\sigma \in S_{\beta}$. Therefore our group 
contains the subgroup $S_{\beta} \times S_{\infty}(\beta)$, which is maximal in $S_{\infty}$. Therefore $G \supset S_{\infty}$. But $S_{\infty}$ and $\mathbb{G}(\beta)$ generate $\mathbb{G}$; see [27, Lemma 3.6].

Proof of Proposition 5.1. Denote by

- $G^{\bigcirc}$ the group generated by $\mathbb{G}(m)$ and $X_{\alpha}^{\bigcirc}(b, c)$;

- $G$ the group generated by $\mathbb{G}(m)$ and $X_{\alpha}^{\square}(b, c)$;

- $G$ the group generated by $\mathbb{G}(\alpha)$ and the matrix $X_{\diamond}(b, c)$ defined by

$$
X_{\diamond}(b, c):=\left(\begin{array}{cc|cc}
1_{\alpha} & 0 & b & 0 \\
0 & 1_{m-\alpha} & 0 & 0 \\
\hline 0 & 0 & 1 & 0 \\
c & 0 & 0 & 1_{\star}
\end{array}\right) .
$$

Obviously, $G \supset G^{\bigcirc}, G \supset G_{\square}$. Let us verify the opposite inclusions.

The inclusion $G^{\bigcirc} \supset G$. Clearly $X_{\alpha}(-b,-c) \in G^{\bigcirc}$. Therefore $G^{\bigcirc}$ contains

$$
X_{\alpha}(b, c) X_{\alpha}(-b,-c)=X\left(\left(\begin{array}{ll}
0 & 0 \\
0 & 2
\end{array}\right),\left(\begin{array}{ll}
0 & 0 \\
0 & 2
\end{array}\right)\right) \sim X\left(\left(\begin{array}{ll}
0 & 0 \\
0 & 1
\end{array}\right),\left(\begin{array}{ll}
0 & 0 \\
0 & 1
\end{array}\right)\right)=: Y .
$$

By Lemma 5.5, the group generated by $Y$ and $\mathbb{G}(m)$ is $\mathbb{G}(\alpha)$. On the other hand, $Y^{-1} X^{\bigcirc}(b, c) \sim X_{\diamond}(b, c)$.

5.3.1. The inclusion $G^{\square} \supset G$. We have

$$
X_{\alpha}^{\square}(b, c)^{2} \sim X_{\diamond}(2 b, 2 c) \sim X_{\diamond}(b, c) .
$$

Next, $X_{\diamond}(b, c)^{-1} X_{\alpha}^{\square}(b, c) \sim X_{\alpha}(0,0)$ and we refer to Lemma 5.6.

Thus, $G^{\bigcirc}=G^{\square}$. By Lemma 5.4, for any unitary representation $\rho$ of $\mathbb{G}$ we have

$$
\left.\left.\widetilde{\rho}_{m m}\left(X^{\bigcirc}(b, c)\right)\right)=\widetilde{\rho}_{m m}\left(X^{\square}(b, c)\right)\right)
$$

and this completes the proof of Proposition 5.1 .

\section{The semigroup $\operatorname{red}_{m}(\Gamma(m))$}

6.1. Structure of the semigroup $\operatorname{red}_{m}(\Gamma(m))$. Denote by $\mathbf{0}$ the minimal idempotent of the semigroup $\operatorname{red}_{m}(\Gamma(m))$.

Proposition 6.1. Any element $\neq \mathbf{0}$ in $\operatorname{red}_{m}(\Gamma(m))$ has a representative of a form $a X(b, c)$, where $a \in \mathrm{GL}(m)$.

The proof occupies the rest of the section. As a byproduct of Lemma 6.3 we will get the following statement.

Lemma 6.2. Any idempotent $[X(b, c)]$ by a conjugation by $a \in \mathrm{GL}(m)$ can be reduced to a form

$$
\left[X\left(\left(\begin{array}{ll}
0 & \beta \\
1 & 0
\end{array}\right),\left(\begin{array}{ll}
0 & 1 \\
\gamma & 0
\end{array}\right)\right)\right]
$$

where $\gamma \beta=0(\bmod p), \beta \gamma=0(\bmod p)$. 


\subsection{Proof of Proposition 6.1.}

Step 1.

\section{Lemma 6.3.}

(a) Let $B$ be an $m \times N$ matrix over $\mathbb{Z}_{p^{\mu}}, C$ an $N \times m$ matrix. Then transformations

$$
B \mapsto u^{-1} B v, \quad C \mapsto v^{-1} C u
$$

allow to reduce them to the form

$$
\widetilde{B}=\left(\begin{array}{cc}
0 & b_{12} \\
b_{21} & b_{22}
\end{array}\right), \quad \widetilde{C}=\left(\begin{array}{cc}
0 & c_{12} \\
c_{21} & c_{22}
\end{array}\right),
$$

where $b_{12}, c_{21}$ are square nondegenerate matrices of the same size, products $b_{21} c_{12}$, $c_{12} b_{21}$ are nilpotent and $b_{22}=0(\bmod p), c_{22}=0(\bmod p)$.

(b) The transformations

$$
B \mapsto u^{-1} B v, \quad C \mapsto w^{-1} C u,
$$

where $u, v, w$ are invertible, allow to reduce a pair $(B, C)$ to the form

$$
\widetilde{B}=\left(\begin{array}{cc}
0 & b_{12} \\
1 & 0
\end{array}\right), \quad \widetilde{C}=\left(\begin{array}{cc}
0 & 1 \\
c_{21} & 0
\end{array}\right),
$$

where $c_{21} b_{12}=0(\bmod p), b_{12} c_{21}=0(\bmod p)$.

Proof. (a) Reduce our matrices modulo $p$. A canonical form of a pair of counter operators $P: \mathbb{F}_{p}^{m} \rightarrow \mathbb{F}_{p}^{N}$ and $Q: \mathbb{F}_{p}^{N} \rightarrow \mathbb{F}_{p}^{m}$ is a standard problem of linear algebra; see, e.g., [7, [1]. In particular, such operators in some bases admit block decompositions $P=\left(\begin{array}{cc}P_{r} & 0 \\ 0 & P_{n}\end{array}\right), Q=\left(\begin{array}{cc}Q_{r} & 0 \\ 0 & Q_{n}\end{array}\right)$, where $P_{r} Q_{r}, Q_{r} P_{r}$ are nondegenerate and $P_{n} Q_{n}, Q_{n} P_{n}$ are nilpotent.

Thus the matrices $B, C$ can be reduced to the form

$$
B^{\prime}=\left(\begin{array}{ll}
b_{11} & b_{12} \\
b_{21} & b_{22}
\end{array}\right), \quad C^{\prime}=\left(\begin{array}{ll}
c_{11} & c_{12} \\
c_{21} & c_{22}
\end{array}\right)
$$

where

(1) $b_{21}, c_{12}$ are invertible matrices of the same size;

(2) products $b_{12} c_{21}, c_{21} b_{12}$ are nilpotent;

(3) the matrices $b_{11}, b_{22}, c_{11}, c_{22}$ reduced $(\bmod p)$ are zero.

Set

$$
u_{1}:=\left(\begin{array}{cc}
1 & b_{11} b_{12}^{-1} \\
0 & 1
\end{array}\right),
$$

notice that $u_{1}(\bmod p)$ is 1 . We pass to new matrices

$$
B^{\prime \prime}=u_{1}^{-1} B^{\prime}, \quad C^{\prime \prime}=C^{\prime \prime} u_{1} .
$$

For new $B$ the block $b_{11}=0$; other properties (1)-(3) of matrices $B, C$ are preserved. Next, we take a unique matrix of the form $u_{2}=\left(\begin{array}{cc}1 & 0 \\ * & 1\end{array}\right)$ such that $C^{\prime \prime} u_{2}$ has zero block $c_{12}$. On the other hand the block $b_{11}$ of $u_{2}^{-1} B^{\prime \prime}$ is zero. We come to a desired form.

(b) We apply statement (a) and reduce $(B, C)$ to the form (6.1). Next, we multiply $\widetilde{B}$ from right by $\left(\begin{array}{ll}b_{21} & \\ & 1\end{array}\right)^{-1}$ and get 1 on the place of $b_{21}$. After this, we 
multiply new $B$ from right by $\left(\begin{array}{cc}1 & -b_{22} \\ 0 & 1\end{array}\right)$ and kill $b_{22}$. Finally, we repeat the same transformations with $\widetilde{C}$.

Now the problem is reduced to the same question for a pair $b_{12}, c_{21}$. If $c_{21} b_{12} \neq$ $0(\bmod p)$, then we choose an invertible matrix $U$ such that $b_{12} U c_{21}$ is not nilpotent and again repeat (a). Etc.

Step 2.

Lemma 6.4. Let $[g] \in \Gamma(m)$ have the form

$$
[g]=\left[\begin{array}{c|c}
1 & b \\
\hline c & 1_{\star}
\end{array}\right]_{m m}
$$

and $[[g]] \approx_{m} \mathbf{0}$. Then bc and $c b$ are nilpotent.

Proof. We apply the previous lemma and represent $[g]$ as

$$
[g]=\left[\begin{array}{cc|cc}
1_{\alpha} & 0 & 0 & b_{12} \\
0 & 1_{m-\alpha} & b_{21} & b_{22} \\
\hline 0 & c_{12} & 1_{m-\alpha} & 0 \\
c_{21} & c_{22} & 0 & 1_{\star}
\end{array}\right]_{m m} .
$$

Set

Let us show that

$$
\left[h_{m}^{\alpha}\right]:=\left[\begin{array}{cc|cc}
1_{\alpha} & 0 & 0 & 0 \\
0 & 1_{m-\alpha} & 1_{m-\alpha} & 0 \\
\hline 0 & 0 & 1_{m-\alpha} & 0 \\
0 & 1_{m-\alpha} & 0 & 1_{m-\alpha \star}
\end{array}\right] .
$$

$$
[g] \circ\left[h_{m}^{\alpha}\right] \sim[g]
$$

Indeed,

$$
\begin{aligned}
{[g] \circ\left[h_{m}^{\alpha}\right]=} & {\left[\begin{array}{cc|cccc}
1_{\alpha} & 0 & 0 & b_{12} & 0 & 0 \\
0 & 1_{m-\alpha} & b_{21} & b_{22} & 1 & 0 \\
\hline 0 & c_{12} & 1_{m-\alpha} & 0 & c_{12} & 0 \\
c_{21} & c_{22} & 0 & 1 & c_{22} & 0 \\
0 & 0 & 0 & 0 & 1 & 0 \\
0 & 1 & 0 & 0 & 0 & 1_{\star}
\end{array}\right]_{m m} } \\
& \sim\left[\begin{array}{cc|cccc|}
1_{\alpha} & 0 & 0 & b_{12} & 0 & 0 \\
0 & 1_{m-\alpha} & b_{21} & b_{22} & 1_{m-\alpha} & 0 \\
\hline 0 & c_{12} & 1_{m-\alpha} & 0 & 0 & 0 \\
c_{21} & c_{22} & 0 & 1 & 0 & 0 \\
0 & 0 & 0 & 0 & 1 & 0 \\
0 & 1_{m-\alpha} & 0 & 0 & 0 & 1_{\star}
\end{array}\right]_{m m}=: r, r
\end{aligned}
$$

to establish the equivalence we multiply $[g] \circ\left[h_{m}^{\alpha}\right]$ from the left by

$$
\left(\begin{array}{c|cccc}
1_{m} & & & & \\
\hline & 1 & 0 & -c_{12} & \\
& 0 & 1 & -c_{22} & \\
& 0 & 0 & 1 & \\
& & & & 1_{\star}
\end{array}\right) .
$$


Next, denote

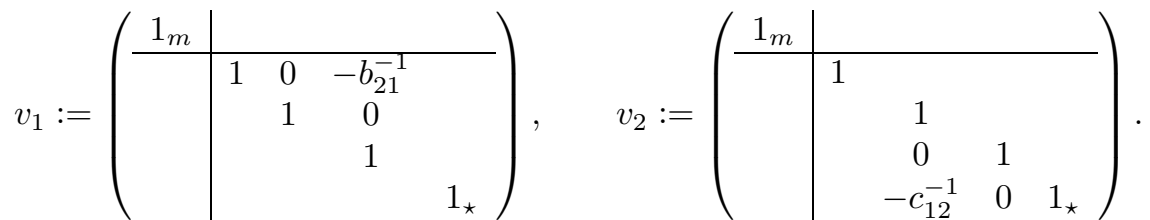

We have

$$
[r] \sim\left[v_{2} v_{1}^{-1} r v_{1} v_{2}^{-1}\right]
$$

the latter matrix is obtained from $r$, see (6.3), by removing two boxed blocks $1_{m-\alpha}$; all other blocks are the same. Thus $[r] \sim[g]$, i.e., we established (6.2).

Suppose that $\alpha \neq m$. Then by Proposition 5.1 .

$$
[g] \sim[g] \circ\left[h_{m}^{\alpha}\right] \approx[g] \circ \Theta_{[m]}^{\alpha} .
$$

But $\Theta_{[m]}^{\alpha} \approx_{m} \mathbf{0}$; therefore $[[g]] \approx_{m} \mathbf{0}$.

Step 3. Thus it is sufficient to prove Proposition 6.1 for $[g]$ having the form

$$
[g]=\left[\begin{array}{c|c}
1 & b \\
\hline c & 1_{\star}
\end{array}\right]_{m m}, \quad \text { where } b c, c b \text { are nilpotent. }
$$

Lemma 6.5. Let $[g]=\left[\begin{array}{c|c}1 & b \\ \hline c & 1_{\star}\end{array}\right]_{m m}$ be invertible 10 Then

$$
\left[g^{-1}\right] \circ[g] \equiv X(b, c) \text {. }
$$

Proof. By (3.1),

$$
[g] \circ\left(\left[g^{-1}\right] \circ[g]\right) \equiv[g], \quad \text { and }\left[g^{-1}\right] \circ[g] \text { is an idempotent. }
$$

We have (see, e.g., [9, Sect. 2.5])

$$
\left[g^{-1}\right]=\left[\begin{array}{c|c}
(1-b c)^{-1} & -(1-b c)^{-1} b \\
\hline-c(1-b c)^{-1} & (1-c b)_{\star}^{-1}
\end{array}\right]_{m m} \sim\left[\begin{array}{c|c}
(1-b c)^{-1} & b \\
\hline c(1-b c)^{-1} & 1_{\star}
\end{array}\right]_{m m} .
$$

We also keep in mind the identity

$$
c(1-b c)^{-1}=(1-c b)^{-1} c,
$$

to establish it, we multiply both sides from the left by $(1-c b)$ and from the right by $(1-b c)$.

Next,

$$
\left[g^{-1}\right] \circ[g]=\left[\begin{array}{c|cc}
(1-b c)^{-1} & b & (1-b c)^{-1} b \\
\hline c(1-b c)^{-1} & 1 & c(1-b c)^{-1} b \\
c & 0 & 1_{\star}
\end{array}\right]_{m m} \sim\left[\begin{array}{c|cc}
(1-b c)^{-1} & b & b \\
\hline c & 1 & 0 \\
c(1-b c)^{-1} & 0 & 1_{\star}
\end{array}\right]_{m m} .
$$

This matrix defines an idempotent in $\operatorname{inv}\left(\Gamma^{\circ}(m)\right)$. We must verify the following statement:

Lemma 6.6. Under our conditions,

$$
\left[g^{-1}\right] \circ[g] \equiv X(b, c) .
$$

\footnotetext{
${ }^{10}$ This is equivalent to invertibility of $(1-b c)^{-1}$ or invertibility of $(1-c b)^{-1}$. Here we do not need a nilpotency of $b c$.
} 
Proof. By Corollary 4.9 we can identify an idempotent in $\operatorname{inv}\left(\Gamma^{\circ}\right)$ evaluating its image in $\operatorname{Mor}_{\mathcal{L}}(m)$. So we get

$$
[g] \circ\left[g^{-1}\right] \equiv[[X(B, C)]],
$$

where

$$
B:=\left(\begin{array}{ll}
b & b
\end{array}\right), \quad C:=\left(\begin{array}{c}
c \\
(1-c b)^{-1} c
\end{array}\right) .
$$

We have $\operatorname{ker} B=\operatorname{ker} b, \operatorname{ker} C^{t}=\operatorname{ker} c^{t}$; therefore by Lemma 4.6 we have $[[X(B, C)]]$ $\equiv[[X(b, c)]]$.

Corollary 6.7. Let

$$
[g]=\left[\begin{array}{c|c}
1 & b \\
\hline c & 1_{\star}
\end{array}\right]_{m m}, \quad\left[g^{\prime}\right]=\left[\begin{array}{l|l}
1 & b u \\
\hline c & 1_{\star}
\end{array}\right]_{m m}
$$

be invertible and $u$ also be invertible. Then

$$
\left[g^{-1}\right] \circ[g] \equiv\left[\left(g^{\prime}\right)^{-1}\right] \circ\left[g^{\prime}\right] .
$$

Proof. Indeed, ker $b u=\operatorname{ker} b$. So both sides are $[[X(b, c)]]$.

Step 4.

Lemma 6.8. Let $[g]=\left[\begin{array}{c|c}1 & b \\ \hline c & 1_{\star}\end{array}\right]_{m m}$; let $b c$ and $c b$ be nilpotent. Then there exists $u$ having the form

$$
u=-\frac{1}{2}+\sum_{j>0} \frac{\sigma_{j}}{2^{n_{j}}}(c b)^{j}, \quad \text { where } \sigma_{j} \in \mathbb{Z}, n_{j} \in \mathbb{Z}_{+},
$$

such that

$$
\begin{aligned}
\left(\left[\left(\begin{array}{l|l}
1 & b u \\
\hline c & 1_{\star}
\end{array}\right)^{-1}\right]_{m m} \circ\left[\begin{array}{l|l}
1 & b u \\
\hline c & 1_{\star}
\end{array}\right]_{m m}\right) \circ\left[g^{-1}\right] \\
\equiv\left[\begin{array}{c|cc}
(1-b u c)^{-1}(1-b c)^{-1} & b & 0 \\
\hline 0 & 1 & 0 \\
c & 0 & 1_{\star}
\end{array}\right]_{m m} .
\end{aligned}
$$

Proof. The product is

$$
\begin{aligned}
& {\left[\begin{array}{c|ccc}
(1-b u c)^{-1}(1-b c)^{-1} & b u & b u & (1-b u c)^{-1} b \\
\hline c(1-b c)^{-1} & 1 & 0 & c b \\
c(1-b u c)^{-1} & 0 & 1 & c(1-b u c)^{-1} \\
c(1-b c)^{-1} & 0 & 0 & 1_{\star}
\end{array}\right]_{m m}} \\
& \sim\left[\begin{array}{c|ccc}
(1-b u c)^{-1}(1-b c)^{-1} & b u & b u & (1-b u c)^{-1} b \\
\hline c & 1 & 0 & 0 \\
c(1-b u c)^{-1} & 0 & 1 & 0 \\
c(1-b c)^{-1} & 0 & 0 & 1_{\star}
\end{array}\right]_{m m}=:\left[\begin{array}{c|c}
A & b r \\
\hline q c & 1_{\star}
\end{array}\right]_{m m},
\end{aligned}
$$

here

$$
r:=\left(\begin{array}{lll}
u & u & (1-c b u)^{-1}
\end{array}\right), \quad q:=\left(\begin{array}{c}
1 \\
(1-c b u)^{-1} \\
(1-c b)^{-1}
\end{array}\right) .
$$

We claim that there exists a unique $u$ such that $r q=0$. A straightforward calcula- 
tion shows that

$$
r q=2 u-u c b u+(1-c b)^{-1} .
$$

Since $c b$ is nilpotent, we can write the equation $r q=0$ as

$$
2 u+1=u c b u-\sum_{j>0}(c b)^{j},
$$

the sum actually is finite. Clearly we can find a solution in the form $u=-1 / 2+$ $\sum_{j>0} s_{j}(c b)^{j}$, where $s_{j}$ are dyadic rationals; for coefficients $s_{j}$ we have a system of recurrent equations. This $u$ is invertible (since we can write a finite series for $u^{-1}$ ).

Next, we must show that the matrix $\left(\begin{array}{l|l}1 & b u \\ \hline c & 1_{\star}\end{array}\right)$ is invertible. Indeed, this is equivalent to existence of $(1-c b u)^{-1}$ and this is clear since by (6.5) $c b u$ is nilpotent.

Next we wish to simplify the matrix $\left(\begin{array}{c|c}A & b r \\ \hline q c & 1_{\star}\end{array}\right)$ by conjugations by matrices of the form $\left(\begin{array}{cc}1 & 0 \\ 0 & D_{\star}\end{array}\right)$. In fact, we have transformations

$$
r \mapsto r^{\prime}=p D^{-1}, \quad q \mapsto q^{\prime}=D q .
$$

For such transformations we have $r^{\prime} q^{\prime}=r q$. Set

$$
D=\left(\begin{array}{ccc}
1 & 1 & u^{-1}(1-c b u)^{-1} \\
0 & 1 & 0 \\
0 & 0 & 1_{\star}
\end{array}\right)
$$

Then $r^{\prime}=\left(\begin{array}{lll}u & 0 & 0\end{array}\right)$. But $u$ is invertible and $r^{\prime} q^{\prime}=0$. Therefore $q^{\prime}$ has the form $\left(\begin{array}{l}0 \\ * \\ *\end{array}\right)$; on the other hand multiplication $q \mapsto D q$ does not change the second and third elements of the column $q$. Thus we came to the matrix

$$
R:=\left[\begin{array}{c|rcc}
(1-b u c)^{-1}(1-b c)^{-1} & b[u & 0 & 0 \\
\hline 0 & 1 & 0 & 0 \\
(1-c b u)^{-1} c & 0 & 1 & 0 \\
\hline(1-c b)^{-1} c & 0 & 0 & 1_{\star}
\end{array}\right]_{m m} .
$$

Consider the following matrices:

$$
S:=\left[\begin{array}{c|ccc}
1 & & & \\
\hline & u & & \\
& & 1-c b u & \\
& & & (1-c b)_{\star}
\end{array}\right], \quad T:=\left[\begin{array}{c|ccc}
1 & & & \\
\hline & 1 & & \\
& 1 & \\
& & -1 & 1_{\star}
\end{array}\right] .
$$

The conjugation $R \mapsto T R T^{-1}$ kills boxed elements of $R$. The conjugation $R \mapsto$ $S T R T^{-1} S^{-1}$ reduces the matrix to the desired form.

Proof. Proof of Proposition 6.1 Thus we have

$$
\left[\left(\begin{array}{c|c}
1 & b \\
\hline c & 1_{\star}
\end{array}\right)^{-1}\right]_{m m} \equiv(1-b u c)^{-1}(1-b c)^{-1} \cdot[[X((1-b c)(1-b u c) b, c)]] .
$$


The second factor is

$$
[[X(b(1-c b)(1-c b u), c)]] \equiv[[X(b, c)]] .
$$

Passing to adjoint elements we get

$$
\begin{aligned}
& {\left[\begin{array}{l|l}
1 & b \\
\hline c & 1
\end{array}\right]_{m m} \equiv[[X(b, c)]] \cdot(1-b c)(1-b u c)} \\
& \quad \equiv(1-b c)(1-b u c) \cdot\left[\left[X\left((1-b u c)^{-1}(1-b c)^{-1} b, c(1-b c)(1-b u c)\right)\right]\right] \\
& \quad \equiv(1-b c)(1-b u c) \cdot[[X(b, c)]] .
\end{aligned}
$$

It remains to notice that

$$
\left[\begin{array}{c|c}
a & b \\
\hline c & 1
\end{array}\right]=a \cdot\left[\begin{array}{c|c}
1 & a^{-1} b \\
\hline c & 1
\end{array}\right] .
$$

\subsection{Proof of Lemma 6.2, We refer to Lemma 6.3.}

\section{The groups $\mathbb{G}^{\bullet}[L ; M]$}

In this section we examine subgroups $\mathbb{G}^{\circ}[L ; M], \mathbb{G}^{\bullet}[L ; M] \subset \mathbb{G}$ defined in Subsection 1.3 . We prove that $\mathbb{G}^{\bullet}[L ; M]$ is well-defined. Lemma 7.5 shows that it is generated by $\mathbb{G}(m)$ and the element $X(b, c)$. Also we prove that it is a minimal subgroup of finite index in $\mathbb{G}[L ; M]$ (equivalently, $\mathbb{G}^{\bullet}[L ; M]$ has no subgroups of finite index, Proposition (7.11).

\subsection{Several remarks on submodules in $\mathfrak{l}^{k}$.}

Lemma 7.1. Let $L \subset \mathfrak{l}^{k}$ be a submodule. Then there exists a basis $e_{j} \in \mathfrak{l}^{k}$ such that $M:=\oplus p^{s_{j}} \mathbb{Z}_{p^{\mu}} e_{j}$. The collection $s_{1}, s_{2}, \ldots$ is a unique $\operatorname{GL}(m)$-invariant of a submodule $L$.

This is equivalent to a classification of sublattices in $\left(\mathbb{O}_{p}\right)^{k}$ under the action of $\operatorname{GL}\left(k, \mathbb{O}_{p}\right)$ or equivalently to a classification of pairs of lattices in $\mathbb{Q}_{p}^{k}$ under $\operatorname{GL}\left(k, \mathbb{Q}_{p}\right)$; the latter question is standard; see, e.g., [35, Theorem I.2.2].

Corollary 7.2. Any submodule $L \subset \mathfrak{l}^{k}$ is a kernel of some endomorphism $\mathfrak{l}^{k} \rightarrow \mathfrak{l}^{k}$.

Indeed, we pass to a canonical basis $e_{j}$ as in the lemma and consider the map sending $e_{j}$ to $p^{\mu-s_{j}} e_{j}$.

\section{Lemma 7.3.}

(a) Let $L$ be a submodule in $\mathfrak{l}^{m}$. Let $b, b^{\prime}: \mathfrak{l}^{m} \rightarrow \mathfrak{l}^{N}$ be morphisms of modules such that $L=\operatorname{ker} b=\operatorname{ker} b^{\prime}$. Then there is a transformation $u \in \operatorname{GL}(N)$ such that $b^{\prime}=b u$.

(b) Let $\operatorname{ker} b=L, \operatorname{ker} b^{\prime}=L^{\prime} \supset L$. Then there is an endomorphism $u: \mathfrak{l}^{N} \rightarrow \mathfrak{l}^{N}$ such that $b^{\prime}=b u$.

Proof. (a) The modules $\operatorname{im} b \simeq \operatorname{im} b^{\prime} \simeq \mathfrak{l}^{m} / L$ are isomorphic. By the previous lemma there is an automorphism of $\mathfrak{l}^{N}$ identifying these submodules.

(b) $L$ is a submodule of $L^{\prime}$; therefore $\operatorname{im} b^{\prime}$ is a quotient module of $\operatorname{im} b$. Therefore there is a projection map $\pi: \operatorname{im} b \rightarrow \operatorname{im} b^{\prime}$; orders of elements do not increase under this map. By Lemma 7.1 we have a basis $e_{j} \in \mathfrak{l}^{N}$ such that $p^{s_{j}} e_{j}$, where $j=1$, $\ldots, m$, is the system of generators of $\operatorname{im} b$. Choose arbitrary vectors $v_{j}$ such that $p^{s_{j}} v_{j}=\pi\left(p^{s_{j}} e_{j}\right)$ and consider the map sending $e_{j}$ to $v_{j}$. 
7.2. The group $\mathbb{G}^{\bullet}$. Here we show that $\mathbb{G}^{\bullet}[L ; M]$ is a group, and its definition does not depend on the choice of matrices $b, c$.

\section{Lemma 7.4.}

(a) Fix a matrix $B$ of size $l \times N$. Then the set of invertible matrices $g$ of the form $1-B S$, where $S$ ranges in the set of $N \times l$ matrices, is a group.

(b) Fix matrices $B, C$ of sizes $l \times N$ and $N \times l$ respectively. Then the set of invertible matrices $g$ of the form $g=1-B u C$ is a group.

Proof. Clearly, both sets are closed with respect to multiplication. We must show that $g^{-1}$ satisfies the same property. In the first case,

$$
1-g^{-1}=1-(1-B S)^{-1}=-B S(1-B S)^{-1} .
$$

In the second case,

$$
1-g^{-1}=1-(1-B u C)^{-1}=-B u C(1-B u C)^{-1}=-B u(1-C B u)^{-1} C .
$$

Lemma 7.5. Fix matrices $b$, $c$ of sizes $m \times N$ and $N \times m$ respectively.

(a) The set of invertible matrices $g=\left(\begin{array}{cc}a & b v \\ w c & z\end{array}\right)$ such that the block ' $a$ ' admits representations $a=1-b S, a=1-T c$ is a group.

(b) The set $\mathbb{G}^{\bullet}[L ; M]$, i.e., the set of all invertible matrices of the form $g=$ $\left(\begin{array}{cc}1-b u c & b v \\ w c & z\end{array}\right)$, is a group.

Proof. In the first case we write

$$
\begin{aligned}
g=\left(\begin{array}{cc}
1-b S & b v \\
w c & z
\end{array}\right)=\left(\begin{array}{ll}
1 & \\
& 1
\end{array}\right)-\left(\begin{array}{cc}
b S & -b v \\
-w c & 1-z
\end{array}\right) \\
\quad=\left(\begin{array}{ll}
1 & \\
& 1
\end{array}\right)-\left(\begin{array}{ll}
b & \\
& 1
\end{array}\right)\left(\begin{array}{cc}
S & -v \\
-w c & 1-z
\end{array}\right),
\end{aligned}
$$

and reduce the statement to the previous lemma.

In the second case we write

$$
g=\left(\begin{array}{ll}
1 & \\
& 1
\end{array}\right)-\left(\begin{array}{ll}
b & \\
& 1
\end{array}\right)\left(\begin{array}{cc}
u & -v \\
-w & 1-z
\end{array}\right)\left(\begin{array}{ll}
c & \\
& 1
\end{array}\right),
$$

and again we apply the previous lemma.

7.3. The group $\mathbb{G}^{\circ}[L ; M]$.

Proof of Lemma 1.3. Let $g=\left(\begin{array}{ll}\alpha & \beta \\ \gamma & \delta_{\star}\end{array}\right) \in \mathbb{G}^{\circ}[L, M]$, i.e., $g$ fix pointwise $L \subset \mathfrak{l}^{m}$ and $g^{t}$ fix pointwise of $M \subset \mathfrak{l}^{m}$. Then $L \subset \operatorname{ker} \beta$ and by Lemma 7.3(b) we have $\beta=b v$ for some matrix $v$. Also $L \subset \operatorname{ker}(1-\alpha)$ and therefore $\alpha=1-b S$ for some $S$.

\subsection{Changes of coordinates.}

Lemma 7.6. Let $L, M \subset \mathfrak{l}^{m}$. Let $a \in \mathrm{GL}(m)$. Then

$$
a \mathbb{G}^{\circ}[L ; M] a^{-1}=\mathbb{G}^{\circ}\left[a L,\left(a^{t}\right)^{-1} M\right], \quad a \mathbb{G}^{\bullet}[L ; M] a^{-1}=\mathbb{G}^{\bullet}\left[a L,\left(a^{t}\right)^{-1} M\right] .
$$

The first statement is an immediate consequence of the definition; the second is straightforward. 
7.5. Generators of $\mathbb{G}^{\bullet}[L ; M]$. Let $m, b, c$ be as in Subsection [1.3, i.e., $L=\operatorname{ker} b$, $M=\operatorname{ker} c^{t} \subset \mathfrak{l}^{m}$.

Proposition 7.7. The group $\mathbb{G} \bullet[L ; M]$ is generated by $\mathbb{G}(m)$ and the matrix $X(b, c)$.

Proof. Consider the group $G$ generated by $\mathbb{G}(m)$ and $X(b, c)$. Clearly, $\mathbb{G}^{\bullet}[L, M] \supset$ $G$. Let us prove the converse.

(1) Conjugating $X(b, c)$ by block diagonal matrices $\in \mathbb{G}(m)$ we get arbitrary matrices of the form $X(b v, w c)$, where $v, w$ are invertible matrices. Consider products

$$
X(b v, w c) X\left(b^{\prime} v, w c^{\prime}\right)=X\left(\left(b+b^{\prime}\right) v, w\left(c+c^{\prime}\right)\right) .
$$

We set $b=-b^{\prime}$; for any matrix $\sigma$ we can find invertible matrices $c, c^{\prime}$ such that 11 $c+c^{\prime}=\sigma$. Thus $G$ contains all matrices of the form

$$
\left(\begin{array}{cc}
1_{m} & b v \\
& 1_{m \star}
\end{array}\right), \quad\left(\begin{array}{cc}
1_{m} & \\
w c & 1_{m \star}
\end{array}\right),
$$

where $v, w$ are arbitrary matrices.

(2) In virtue of Lemma 6.2, conjugating the matrices (7.2) by elements of GL $(m)$ and multiplying from the left and the right by elements of $\mathbb{G}(m)$ we can reduce the matrices (7.2) to the forms

$$
Y[\beta]:=\left(\begin{array}{cccc}
1_{m-\alpha} & 0 & 0 & \beta \\
0 & 1_{\alpha} & 1_{\alpha} & 0 \\
0 & 0 & 1_{\alpha} & 0 \\
0 & 0 & 0 & 1_{m-\alpha \star}
\end{array}\right), Z[\gamma]:=\left(\begin{array}{cccc}
1_{m-\alpha} & 0 & 0 & 0 \\
0 & 1_{\alpha} & 0 & 0 \\
0 & 1_{\alpha} & 1_{\alpha} & 0 \\
\gamma & 0 & 0 & 1_{m-\alpha \star}
\end{array}\right),
$$

where $\gamma \beta=0(\bmod p), \beta \gamma=0(\bmod p)$. Multiplying $Y[\beta]$ from right by elements of $\mathbb{G}(m+\alpha)$ we can get any matrix $Y[\beta r]$ with invertible $r$. The condition of invertibility of $r$ can be removed, because

$$
Y\left[\beta r_{1}\right] Y\left[\beta r_{2}\right]^{-1} Y\left[\beta r_{3}\right]=Y\left[\beta\left(r_{1}-r_{2}+r_{3}\right)\right],
$$

and we can represent any matrix $r$ as a sum of 3 invertible matrices.

In the same way we get that $G$ contains all elements of the form $Z[q \gamma]$.

Take $r=0, q=0$. Then the matrices $Y[0]=Y[\beta \cdot 0], Z[0]=Z[0 \cdot \gamma]$ together with $\mathbb{G}(m)$ generate the group $\mathbb{G}(m-\alpha)$.

Next, $G$ contains matrices $Y[\beta] Y[0]^{-1}$ and $Z[\gamma] Z[0]^{-1}$. They are matrices of the form (7.3), where boxed blocks are replaced by zeroes.

Therefore our problem is reduced to a description of the subgroup generated by $\mathbb{G}(m-\alpha)$ and $X(\beta, \gamma)$.

Thus, without loss of generality, we can assume that $\alpha=0$ and $c b=0(\bmod p)$, $b c=0(\bmod p)$.

(3) Multiplying the matrices (7.2), we get

$$
\left(\begin{array}{cc}
1-b v w c & b v \\
w c & 1_{\star}
\end{array}\right) \in G \text { for any } v, w .
$$

\footnotetext{
${ }^{11}$ It is sufficient to verify this statement for matrices over $\mathbb{F}_{p}$. Without loss of generality we can assume that $\sigma$ is diagonal. For $p \neq 2$ any element of $\mathbb{F}_{p}$ is a sum of two nonzero elements, where $\sigma$ can be represented as a sum of two diagonal matrices.
} 
Since $c b=0(\bmod p)$, the $b v w c$ is nilpotent, and therefore $1-b v w c$ is invertible. We represent our matrix as

$$
\begin{aligned}
\left(\begin{array}{cc}
1 & 0 \\
w c(1-b v w c)^{-1} & 1_{\star}
\end{array}\right) & \left(\begin{array}{cc}
1-b v w c & 0 \\
0 & 1_{\star}
\end{array}\right) \\
\times & \left(\begin{array}{cc}
1 & 0 \\
0 & 1-w c(1-b v w c)^{-1} b v_{\star}
\end{array}\right)\left(\begin{array}{cc}
1 & (1-b v w c)^{-1} b v \\
0 & 1 \star
\end{array}\right) .
\end{aligned}
$$

Since the whole product and three factors are contained in $G$, the fourth factor also is contained in $G$,

$$
\left(\begin{array}{cc}
1-b v w c & 0 \\
0 & 1_{\star}
\end{array}\right) \in G
$$

for any $v, w$.

(4) Now consider an arbitrary element of $\mathbb{G}^{\bullet}[L ; M]$,

$$
\begin{aligned}
\left(\begin{array}{cc}
1-b u c & b v \\
w c & z_{\star}
\end{array}\right)=\left(\begin{array}{cc}
1 & 0 \\
w c(1-b u c)^{-1} & 1_{\star}
\end{array}\right)\left(\begin{array}{cc}
1-b u c & 0 \\
0 & 1_{\star}
\end{array}\right) & \\
\times\left(\begin{array}{cc}
1 & 0 \\
0 & z-w c(1-b u c)^{-1} b v_{\star}
\end{array}\right) & \left(\begin{array}{cc}
1 & (1-b u c)^{-1} b v \\
0 & 1_{\star}
\end{array}\right) .
\end{aligned}
$$

All factors of the right hand side are contained in $G$, and therefore $\mathbb{G}^{\bullet}[L ; M]$ is contained in $G$.

Corollary 7.8. The group $\mathbb{G}^{\bullet}[L ; M]$ does not depend on a choice of $m$.

Proof. Let $L, M \subset \mathfrak{l}^{m}$; let $L=\operatorname{ker} b, M=\operatorname{ker} c^{t}$. Let us regard $L, M$ as submodules $L^{\prime}, M^{\prime}$ of $\mathfrak{l}^{m} \oplus \mathfrak{l}^{k}$. Then

$$
L^{\prime}=\operatorname{ker} b^{\prime}, M^{\prime}=\operatorname{ker}\left(c^{\prime}\right) t, \text { where } b^{\prime}=\left(\begin{array}{ll}
b & 0 \\
0 & 1
\end{array}\right), c^{\prime}=\left(\begin{array}{cc}
c & 0 \\
0 & 1
\end{array}\right) .
$$

Clearly the subgroup $G_{m}$ generated by $\mathbb{G}(m)$ and $X(b, c)$ and the subgroup $G_{m+k}$ generated by $\mathbb{G}(m+k)$ and $X\left(b^{\prime}, c^{\prime}\right)$ coincide. Formally, we must repeat the first two steps of the previous proof.

\subsection{The quotient $\mathbb{G}^{\circ} / \mathbb{G}^{\bullet}$.}

Lemma 7.9. A group $\mathbb{G}^{\bullet}[L ; M]$ has finite index in $\mathbb{G}^{\circ}[L ; M]$.

Proof. Without loss of generality we can assume that $c b=0(\bmod p), b c=0(\bmod p)$. Denote by $A^{\circ} \subset \mathrm{GL}(m)$ the subgroup consisting of matrices $a$ admitting representations $a=1-b S, a=1-T c$. Notice that $1-a$ is a nilpotent, since $T c b S=0(\bmod p)$. Therefore $a$ is invertible. Denote by $A^{\bullet}$ the subgroup consisting of elements of the form $1-b u c$.

The subgroup $A^{\bullet}$ is normal in $A^{\circ}$. Indeed, let $a \in A^{\circ}, a=1-b S, a^{-1}=1-T c$. Then

$a(1-b u c) a^{-1}=1-a b u c a^{-1}=1-(1-b S) b u c(1-T c)=1-b(1-S b) u(1-c T) c$.

Let $g=\left(\begin{array}{cc}a & b v \\ w c & z\end{array}\right) \in \mathbb{G}^{\circ}[L ; M]$. Let us show that the map $g \mapsto a$ induces a homomorphism from $\mathbb{G}^{\circ}[L ; M] \rightarrow A^{\circ} / A^{\bullet}$. Indeed,

$$
g_{1} g_{2}=\left(\begin{array}{cc}
a_{1} & b v_{1} \\
w_{1} & z_{1}
\end{array}\right)\left(\begin{array}{cc}
a_{2} & b v_{2} \\
w_{2} c & z_{2}
\end{array}\right)=\left(\begin{array}{cc}
a_{1} a_{2}+b v_{1} w_{2} c & * \\
* & *
\end{array}\right) .
$$


In the left upper block we have

$$
a_{1} a_{2}\left(1+a_{2}^{-1} a_{1}^{-1} b v_{1} w_{2} c\right) .
$$

We represent $a_{1}^{-1}=1-b S_{1}, a_{2}^{-1}=1-b S_{2}$ and get

$$
a_{1} a_{2}\left(1+\left(1-b S_{2}\right)\left(1-b S_{1}\right) b v_{1} w_{2} c\right)=a_{1} a_{2}\left\{1+b\left(1-S_{2} b\right)\left(1-S_{1} b\right) v_{1} w_{2} c\right\} .
$$

The expression in the curly brackets is contained in $A^{\bullet}$.

Clearly, the kernel of the homomorphism is $\mathbb{G}^{\bullet}[L, M]$. Thus we have an isomorphism of quotient groups,

$$
\mathbb{G}^{\circ}[L ; M] / \mathbb{G}^{\bullet}[L ; M] \simeq A^{\circ}[L ; M] / A^{\bullet}[L ; M] .
$$

The group on the right-hand side is finite.

\subsection{Absence of subgroups of finite index.}

Lemma 7.10. The group $\mathbb{G}$ has not proper open subgroups of finite index.

Proof. Let $P$ be a proper open subgroup. Then it contains some group $\mathbb{G}(\nu)$. On the other hand $\mathbb{G}$ contains a complete infinite symmetric group $S_{\infty}$, and $S_{\infty}$ has no subgroups of finite index. Therefore $P$ contains $S_{\infty}$. But the subgroup in $\mathbb{G}$ generated by $\mathbb{G}(\nu)$ and $S_{\infty}$ is the whole group $\mathbb{G}$; see [27, Lemma 3.6].

Proposition 7.11. The subgroup $\mathbb{G}^{\bullet}[L ; M]$ has no proper open subgroups of finite index.

Proof. Let $Q$ be such subgroup. By the previous lemma, $\mathbb{G}(m)$ has not open subgroups of finite index; we have $Q \supset \mathbb{G}(m)$. Hence $Q$ contains a minimal normal subgroup $R$ containing $\mathbb{G}(m)$. The quotient $Q / R$ is generated by the image $\xi$ of $X(b, c)$; therefore $Q / R$ is a cyclic group. But

$$
X(b, c)^{2}=X(2 b, 2 c)=\left(\begin{array}{l|ll}
1 & & \\
\hline & 1 / 2 & \\
& & 2_{\star}
\end{array}\right)\left(\begin{array}{l|ll}
1 & b & 0 \\
\hline 0 & 1 & 0 \\
c & 0 & 1_{\star}
\end{array}\right)\left(\begin{array}{l|ll}
1 & & \\
\hline & 2 & \\
& & 1 / 2 \star
\end{array}\right) .
$$

Since $p \neq 2$ the elements $X(b, c)^{2}$ and $X(b, c)$ have the same images in $Q / R$. Therefore the image of $X[b, c]$ is 1 .

Corollary 7.12. Any subgroup of finite index in $\mathbb{G}[L, M]$ contains $\mathbb{G} \bullet[L, M]$.

\section{END OF THE PROOF}

This section contains the end of the proof of Theorem 1.5. We know that all idempotents in semigroups $\operatorname{red}(\Gamma(n))$ have the form $\mathcal{X}[L, M]$, see Corollary 5.2 , for different $n$ they can be identified in a natural way; see Proposition 5.3 . We also know that any non-zero element of $\operatorname{red}_{m}(\Gamma(m))$ is a product of an invertible element and an idempotent $\mathcal{X}[L, M]$; see Proposition 6.1. This implies that all irreducible representations of $\mathbb{G}$ are induced from representations $\tau$ of groups $\mathbb{G}[L ; M]$. Proposition 7.11 implies that such $\tau$ must be trivial on $\mathbb{G}^{\bullet}[L ; M]$. 


\subsection{A preliminary remark.}

Lemma 8.1. Consider an irreducible *-representation $\sigma$ of the category $\mathcal{K}$ in a sequence of Hilbert spaces $H_{j}$. Let $\xi \in H_{m}$ be a nonzero vector. Then the matrix element

$$
c(\mathfrak{g})=\langle\sigma(\mathfrak{g}) \xi, \xi\rangle_{H_{m}}, \quad \text { where } \mathfrak{g} \text { ranges in } \operatorname{End}_{\mathcal{K}}(m),
$$

determines $\sigma$ up to equivalence.

This is a general statement on *-representations of categories (and a copy of a similar statement for unitary representations of groups); we give a proof for completeness.

Proof. For each $\mathfrak{g} \in \operatorname{Mor}_{\mathcal{K}}(m, \alpha)$ we define a vector

$$
\omega_{\mathfrak{g}}^{\alpha}=\sigma(\mathfrak{g}) \xi \in H_{\alpha} .
$$

Since $\sigma$ is irreducible, vectors $\omega_{\mathfrak{g}}^{\alpha}$, where $\mathfrak{g}$ ranges in $\operatorname{Mor}_{\mathcal{K}}(m, \alpha)$, generate the space $H_{\alpha}$. Their inner products are determined by the function $c$ :

$$
\left\langle\omega_{\mathfrak{g}_{1}}^{\alpha}, \omega_{\mathfrak{g}_{2}}^{\alpha}\right\rangle_{H_{\alpha}}=\left\langle\sigma\left(\mathfrak{g}_{1}\right) \xi, \sigma\left(\mathfrak{g}_{2}\right) \xi\right\rangle_{H_{\alpha}}=\left\langle\sigma\left(\mathfrak{g}_{2}^{*} \circ \mathfrak{g}_{1}\right) \xi, \xi\right\rangle_{H_{m}}=c\left(\mathfrak{g}_{2}^{*} \circ \mathfrak{g}_{1}\right) .
$$

Next, let $\mathfrak{h} \in \operatorname{Mor}_{\mathcal{K}}(\alpha, \beta)$. Let $\mathfrak{g}, \mathfrak{f}$ range respectively in $\operatorname{Mor}_{\mathcal{K}}(m, \alpha), \operatorname{Mor}_{\mathcal{K}}(m, \beta)$. Then

$$
\left\langle\sigma(\mathfrak{h}) \omega_{\mathfrak{g}}, \omega_{\mathfrak{f}}\right\rangle_{H_{\beta}}=\langle\sigma(\mathfrak{h}) \sigma(\mathfrak{g}) \xi, \sigma(\mathfrak{f}) \xi\rangle_{H_{\beta}}=\left\langle\sigma\left(\mathfrak{f}^{*} \circ \mathfrak{h} \circ \mathfrak{g}\right) \xi, \xi\right\rangle_{H_{m}}=c\left(\mathfrak{f}^{*} \circ \mathfrak{h} \circ \mathfrak{g}\right) .
$$

Clearly an operator $\sigma(\mathfrak{h})$ is uniquely determined by such inner products.

8.2. Representations of the semigroup $\operatorname{red}_{m}(\Gamma(m))$. Consider an irreducible representation of $\mathcal{K}$ of height $m$ and the corresponding representation $\lambda$ of the semigroup $\operatorname{End}_{\mathcal{K}}(m)$ in $H_{m}$. Recall that $\tau$ passes through semigroup $\operatorname{red}_{m}(\Gamma(m))$. By Proposition 6.1, any nonzero element of the latter semigroup can be represented as $a \cdot X[L, M]$, where $a \in \mathrm{GL}(m)$. Denote

$$
\widehat{\mathbb{G}}_{n}[L, M]=\mathrm{GL}(n) \cap \widehat{\mathbb{G}}[L, M], \quad \widehat{\mathbb{G}}_{\mathrm{fin}}[L, M]=\mathbb{G}_{\mathrm{fin}} \cap \widehat{\mathbb{G}}_{m}[L, M] .
$$

Lemma 8.2 is a special case of general description of representations of finite inverse semigroups; see, e.g., [10]. However, due to Proposition 6.1] our case is simpler than general inverse semigroups. We show that the representation of $\operatorname{GL}(m)$ in $H_{m}$ is induced from an irreducible representation of some subgroup $\widehat{\mathbb{G}}_{m}[L, M]$ and idempotents $\mathcal{X}[N, K]$ act in the induced representation as multiplications by indicator functions of certain sets. Precisely,

Lemma 8.2. Let $\mathcal{X}[L, M]$ be the minimal idempotent in $\operatorname{red}_{m}(\Gamma(m))$ such that $\lambda(X[L, M]) \neq 0$. Then there is an irreducible representation $\tau_{m}$ of $\widehat{\mathbb{G}}_{m}[L, M]$ in a space $V$ such that $H_{m}$ can be identified with the space $\ell_{2}$ of $V$-valued functions on the homogeneous space $\widehat{\mathbb{G}}_{m}[L, M] \backslash \mathrm{GL}(m)$ and

(1) The group $\mathrm{GL}(m)$ acts by transformations of the form

$$
\lambda(g) f(x)=R(g, x) f(x g),
$$

and for $q \in \widehat{\mathbb{G}}_{m}[L, M]$ we have $R\left(p, x_{0}\right)=\tau_{m}(q)$ (where $x_{0}$ denotes the initial point of $\left.\widehat{\mathbb{G}}_{m}[L, M] \backslash \mathrm{GL}(m)\right)$. 
(2) The semigroup of idempotents acts by multiplications by indicator functions. Namely $\mathcal{X}[K, N]$ acts by multiplication by the function

$$
I_{K, N}\left(x_{0} a\right)= \begin{cases}1, & \text { if } K \supset a L, N \supset\left(a^{t}\right)^{-1} M ; \\ 0, & \text { otherwise. }\end{cases}
$$

Proof. Consider the image $V$ of the projector $\lambda(\mathcal{X}[L, M])$. The idempotent $\mathcal{X}[L, M]$ commutes with $\widehat{\mathbb{G}}_{m}[L, M]$. Indeed, for $q \in \widehat{\mathbb{G}}_{m}[L, M]$ we have

$$
q \cdot X[L, M] \cdot q^{-1}=X\left[L q, M\left(q^{t}\right)^{-1}\right]=X[L, M] .
$$

Therefore the subspace $V$ is $\widehat{\mathbb{G}}_{m}[L, M]$-invariant. Denote by $\tau_{m}$ the representation of the group $\widehat{\mathbb{G}}_{m}[L, M]$ in $V$. We need Lemma 8.3

Lemma 8.3. For any $\mathfrak{g} \in \operatorname{red}_{m}(\Gamma(m))$ we have $\lambda(\mathfrak{g}) V=V$ or $\lambda(\mathfrak{g}) V \perp V$.

Proof of Lemma 8.3. Let us apply an arbitrary element of $\operatorname{red}_{m}(\Gamma(m))$ to $v \in V$, $\lambda(a \cdot X[K, N]) v=\lambda(a) \cdot \lambda(X[K, N] \mathcal{X}[L, M]) v=\lambda(a) \cdot \lambda(X[K \cap L, N \cap M]) v$.

We have the following cases:

(1) If $K \not \supset L$ or $N \not \supset M$, then by our choice of $\mathcal{X}[L, M]$, we have

$$
\lambda(X[K \cap L, N \cap M])=0 .
$$

(2) Otherwise we come to $\lambda(a) \lambda(\mathcal{X}[L, M]) v=\lambda(a) v$.

(2.1) If $a \in \widehat{\mathbb{G}}_{m}[L, M]$, we get $\lambda(a) v \in V$.

(2.2) Let $a \notin \widehat{\mathbb{G}}_{m}[L, M]$. Then

$$
\begin{array}{r}
\lambda(X[L, M]) \lambda(a) \lambda(X[L, M]) v=\lambda(a)\left\{\lambda\left(a^{-1}\right) \lambda(X[L, M]) \lambda(a)\right\} \lambda(X[L, M]) v \\
=\lambda(a) \lambda\left(X\left[L a, M\left(a^{t}\right)^{-1}\right]\right) \lambda(X[L ; M]) v \\
=\lambda(a) \lambda\left(X\left[L a \cap L, M\left(a^{t}\right)^{-1} \cap M\right]\right) v=0 .
\end{array}
$$

Since an idempotent $\mathcal{X}\left[a^{-1} L \cap L, a^{t} M \cap M\right]$ is strictly smaller than $\mathcal{X}[L, M]$, the $\lambda(X[\ldots])=0$.

End of proof of Lemma 8.2. Thus $H_{m}$ is an orthogonal direct sum of spaces $V_{x}$, where $x$ ranges in the homogeneous space $\widehat{\mathbb{G}}_{m}[L, M] \backslash \mathrm{GL}(m)$, and $\lambda(a)$ sends each $V_{x}$ to $V_{x a}$. This means that the representation $\lambda$ of $\operatorname{GL}(m)$ is induced from the representation of $\widehat{\mathbb{G}}_{m}[L, M]$ in $V$; see, e.g., [33, Sect.7.1].

Operators

$$
\lambda\left(X\left[L a^{-1}, a^{t} M\right]\right)=\lambda(a) \lambda(X[L, M]) \lambda\left(a^{-1}\right)
$$

act as orthogonal projectors to $V_{x_{0} a}$. A projector $\lambda(\mathcal{X}[K, N])$ is identical on $V_{x_{0} a}$ if and only if $\mathcal{X}[K, N] \mathcal{X}\left[L a^{-1}, M a^{t}\right]=X\left[L a^{-1}, M a^{t}\right]$ and this gives us the action of the semigroup of idempotents.

It remains to show the representation of $\widehat{\mathbb{G}}_{m}[L, M]$ in $V$ is irreducible. Assume that it contains a $\widehat{\mathbb{G}}_{m}[L, M]$-invariant subspace $W$; then each $V_{x}$ contains a copy $W_{x}$ of $W$ and $\oplus_{x} W_{x}$ is a GL $(m)$-invariant subspace in the whole $H_{m}$.

Corollary 8.4. Let $\lambda(\mathfrak{g})$ be a nonzero operator leaving $V$ invariant. Then there is $b \in \widehat{\mathbb{G}}_{m}[L, M]$ such that

$$
\left.\lambda(\mathfrak{g})\right|_{V}=\left.\rho(b)\right|_{V}
$$


Proof. This operator can be represented as $\lambda(a) \lambda(\mathcal{X}[N, K])$. An operator $\lambda(\mathcal{X}[N, K])$ restricted to $V$ is 0 or 1 . Let this operator be 1 . Then $\lambda(a)$ preserves $V$ only if $a \in \widehat{\mathbb{G}}_{m}[L, M]$. In this case we set $b=a$.

Keeping in mind Lemma 8.1 we get the following statement:

Corollary 8.5. An irreducible $*$-representation of the category $\mathcal{K}$ is determined by its height $m$, a minimal idempotent $X[L, M]$ acting nontrivially in $H_{m}$ and an irreducible representation $\tau$ of the group $\widehat{\mathbb{G}}_{m}[L, M]$.

We do not claim an existence of representation corresponding to given data of this kind.

8.3. End of proof. Let $\rho$ be an irreducible unitary representation of $\mathbb{G}$ of height $m$ in a Hilbert space $H$. Then we have a chain of subspaces in $H$ :

$$
H_{m} \longrightarrow H_{m+1} \longrightarrow H_{m+2} \longrightarrow \ldots
$$

Lemma 4.2 defines a chain of semigroups

$$
\Gamma(m) \longrightarrow \Gamma(m+1) \longrightarrow \Gamma(m+2) \longrightarrow \ldots
$$

Each semigroup $\Gamma(n)$ acts in $H$ as follows: in $H_{n}$ it acts by operators $\widetilde{\rho}_{n n}(\cdot)$; on $H_{n}^{\perp}$ these operators are zero (see Lemma 4.1).

On the other hand, we have a chain of groups

$$
\mathrm{GL}(m) \longrightarrow \mathrm{GL}(m+1) \longrightarrow \mathrm{GL}(m+2) \longrightarrow \ldots
$$

acting by unitary operators; their inductive limit is the group $\mathbb{G}_{f i n}$. Each group $\mathrm{GL}(n)$ preserves the subspace $H_{n}$; on this subspace the action of $\mathrm{GL}(n)$ coincides with the action of the group $\operatorname{Aut}_{\mathcal{K}}(n)=\mathrm{GL}(n)$.

Consider the data listed in Corollary 8.5. We regard the subspace

$$
V=\operatorname{im} \widetilde{\rho}_{m m}(X[L, M]) \subset H_{m}
$$

as a subspace in $H$. Denote the GL(n)-cyclic of $V$ by $W_{n}$; it is a subspace in $H_{n}$.

Lemma 8.6. Let $g \in \mathrm{GL}(n)$. If $g \in \widetilde{\mathbb{G}}_{n}[L ; M]$, then $\rho(g) V=V$. Otherwise, $\rho(g) V \perp V$.

Proof. In the first case, we have

$$
\widetilde{\rho}_{n n}(X[L, M])\left(\widetilde{\rho}_{n n}(g)\right)^{-1} \widetilde{\rho}_{n n}\left(X\left[L g, M\left(g^{t}\right)^{-1}\right]\right)=\widetilde{\rho}_{n n}(X[L, M])
$$

and therefore the image $V$ of $\widetilde{\rho}_{n n}(\mathcal{X}[L, M])$ is invariant with respect to $\rho(g)$.

In the second case we repeat the line (8.1).

Thus the representation of GL $(n)$ in $W_{n}$ is induced from the subgroup $\widehat{\mathbb{G}}_{n}[L, M]$. If $k>n$, then we have embeddings

$$
\mathrm{GL}(n) \rightarrow \mathrm{GL}(k), \quad \widehat{\mathbb{G}}_{n}[L, M] \rightarrow \widehat{\mathbb{G}}_{k}[L, M]
$$

and therefore the map of homogeneous spaces

$$
\Xi_{n, k}: \widehat{\mathbb{G}}_{n}[L, M] \backslash \mathrm{GL}(n) \rightarrow \widehat{\mathbb{G}}_{k}[L, M] \backslash \mathrm{GL}(k) .
$$

On the other hand, we have an embedding $W_{n} \rightarrow W_{k}$ regarding the orthogonal decompositions of these spaces into copies of $V$; therefore the map $\Xi_{n, k}$ is an embedding. 
Finally, we get a representation of $\mathbb{G}_{\text {fin }}$ induced from the subgroup $\widehat{\mathbb{G}}_{\text {fin }}[L, M]$. By continuity, $\mathbb{G}$ acts regarding the same orthogonal decomposition $\oplus V_{x a}$. Hence a representation of $\mathbb{G}$ is induced from closure 12 of $\widehat{\mathbb{G}}_{\mathrm{fin}}[L, M]$, i.e., $\widehat{\mathbb{G}}[L, M]$.

Lemma 8.7. The image of $\widehat{\mathbb{G}}_{\mathrm{fin}}[L, M]$ in the group of operators in $V$ coincides with the image of $\widehat{\mathbb{G}}_{m}[L, M]$.

Proof. Let $u \in \widehat{\mathbb{G}}_{n}[L, M]$. Then $\rho(u)$ preserves $V \subset H_{m}$. Therefore

$$
\left.\rho(u)\right|_{V}=\left.P_{m} \rho(u) P_{m}\right|_{V}=\left.\widetilde{\rho}\left([u]_{m m}\right)\right|_{V} .
$$

By Corollary 8.4, this operator has the form $\left.\rho\left(u^{\prime}\right)\right|_{V}$, where $u^{\prime} \in \widehat{\mathbb{G}}_{m}[L, M]$.

Thus the representation $\tau$ of $\widehat{\mathbb{G}}_{\text {fin }}[L ; M]$ in $V$ has a finite image. Its continuous extension to $\mathbb{G}[L ; M]$ has the same image. The kernel of the representation $\tau$ is a closed subgroup. Since it has a finite index, it is open. By Proposition [7.11, $\tau$ is trivial on the subgroup $\mathbb{G}^{\bullet}[L ; M]$.

\section{REFERENCES}

[1] David J. Aldous, Exchangeability and related topics, École d'été de probabilités de SaintFlour, XIII-1983, Lecture Notes in Math., vol. 1117, Springer, Berlin, 1985, pp. 1-198, DOI 10.1007/BFb0099421. MR883646

[2] Itaï Ben Yaacov, Tomás Ibarlucía, and Todor Tsankov, Eberlein oligomorphic groups, Trans. Amer. Math. Soc. 370 (2018), no. 3, 2181-2209, DOI 10.1090/tran/7227. MR3739206

[3] Alexei Borodin and Grigori Ol'shanskǐ, Harmonic analysis on the infinite-dimensional unitary group and determinantal point processes, Ann. of Math. (2) 161 (2005), no. 3, 1319-1422, DOI 10.4007/annals.2005.161.1319. MR2180403

[4] Alexander I. Bufetov and Yanqi Qiu, Ergodic measures on spaces of infinite matrices over non-Archimedean locally compact fields, Compos. Math. 153 (2017), no. 12, 2482-2533, DOI 10.1112/S0010437X17007412. MR3705296

[5] Peter J. Cameron, Oligomorphic permutation groups, London Mathematical Society Lecture Note Series, vol. 152, Cambridge University Press, Cambridge, 1990, DOI 10.1017/CBO9780511549809. MR1066691

[6] A. H. Clifford and G. B. Preston, The algebraic theory of semigroups. Vol. I, Mathematical Surveys, No. 7, American Mathematical Society, Providence, R.I., 1961. MR0132791

[7] N. M. Dobrovol'skaja and V. A. Ponomarev, A pair of counter-operators (Russian), Uspehi Mat. Nauk 20 (1965), no. 6, 81-86. MR0219551

[8] A. V. Dudko, Tame representations of the group $\mathrm{GL}\left(\infty, \mathbb{F}_{q}\right)$ (Russian), Algebra i Analiz 18 (2006), no. 2, 57-79, DOI 10.1090/S1061-0022-07-00949-1; English transl., St. Petersburg Math. J. 18 (2007), no. 2, 223-239. MR2244936

[9] F. R. Gantmacher, The theory of matrices. Vol. 1, AMS Chelsea Publishing, Providence, RI, 1998. Translated from the Russian by K. A. Hirsch; Reprint of the 1959 translation. MR 1657129

[10] Olexandr Ganyushkin, Volodymyr Mazorchuk, and Benjamin Steinberg, On the irreducible representations of a finite semigroup, Proc. Amer. Math. Soc. 137 (2009), no. 11, 3585-3592, DOI 10.1090/S0002-9939-09-09857-8. MR2529864

[11] R. A. Horn and V. V. Sergeichuk, Representations of quivers and mixed graphs, Chapter 34 in: L. Hogben (Ed.), Handbook of linear algebra, 2nd ed., Chapman and Hall, CRC, Boca Raton, FL, 2014.

[12] J. Kastl, Inverse categories, Algebraische Modelle, Kategorien und Gruppoide, Stud. Algebra Anwendungen, vol. 7, Akademie-Verlag, Berlin, 1979, pp. 51-60. MR569574

[13] Alexander S. Kechris and Christian Rosendal, Turbulence, amalgamation, and generic automorphisms of homogeneous structures, Proc. Lond. Math. Soc. (3) 94 (2007), no. 2, 302-350, DOI 10.1112/plms/pdl007. MR2308230

\footnotetext{
${ }^{12}$ This closure contains $\mathbb{G}(m)$ and we refer to Lemma 3.2
} 
[14] Sergei Kerov, Grigori Ol'shanskǐ, and Anatoly Vershik, Harmonic analysis on the infinite symmetric group, Invent. Math. 158 (2004), no. 3, 551-642, DOI 10.1007/s00222-004-0381-4. MR2104794

[15] A. A. Kirillov, Elements of the theory of representations, Grundlehren der Mathematischen Wissenschaften, Band 220, Springer-Verlag, Berlin-New York, 1976. Translated from the Russian by Edwin Hewitt. MR0412321

[16] A. G. Kurosh, Obshchaya algebra (Russian), Izdat. "Nauka", Moscow, 1974. Lectures for the academic year 1969-1970; Edited by T. M. Baranovič. MR0392756

[17] Mark V. Lawson, Inverse semigroups, World Scientific Publishing Co., Inc., River Edge, NJ, 1998. The theory of partial symmetries, DOI 10.1142/9789812816689. MR1694900

[18] Maxim Nazarov, Oscillator semigroup over a non-Archimedean field, J. Funct. Anal. 128 (1995), no. 2, 384-438, DOI 10.1006/jfan.1995.1037. MR.1319962

[19] Maxime Nazarov, Yuriǔ Neretin, and Grigoriŭ Ol'shanskǔr, Semi-groupes engendrés par la représentation de Weil du groupe symplectique de dimension infinie (French, with English summary), C. R. Acad. Sci. Paris Sér. I Math. 309 (1989), no. 7, 443-446. MR.1055455

[20] Yu. A. Neretin, Categories of symmetries and infinite-dimensional groups, London Mathematical Society Monographs. New Series, vol. 16, The Clarendon Press, Oxford University Press, New York, 1996. Translated from the Russian by G. G. Gould; Oxford Science Publications. MR.1418863

[21] Yurii A. Neretin, Hua-type integrals over unitary groups and over projective limits of unitary groups, Duke Math. J. 114 (2002), no. 2, 239-266, DOI 10.1215/S0012-7094-02-11423-9. MR.1920189

[22] Yurii A. Neretin, Lectures on Gaussian integral operators and classical groups, EMS Series of Lectures in Mathematics, European Mathematical Society (EMS), Zürich, 2011, DOI 10.4171/080. MR2790054

[23] Yu. A. Neretin, Sphericity and multiplication of double cosets for infinite-dimensional classical groups (Russian, with Russian summary), Funktsional. Anal. i Prilozhen. 45 (2011), no. 3, 79-96, DOI 10.1007/s10688-011-0025-6; English transl., Funct. Anal. Appl. 45 (2011), no. 3, 225-239. MR2883240

[24] Yu. A. Neretin, Hua measures on the space of p-adic matrices and inverse limits of Grassmannians (Russian, with Russian summary), Izv. Ross. Akad. Nauk Ser. Mat. 77 (2013), no. 5, 95-108, DOI 10.1070/im2013v077n05abeh002665; English transl., Izv. Math. 77 (2013), no. 5, 941-953. MR.3137196

[25] Yu. A. Neretin, Infinite-dimensional p-adic groups, semigroups of double cosets, and inner functions on Bruhat-Tits buildings (Russian, with Russian summary), Izv. Ross. Akad. Nauk Ser. Mat. 79 (2015), no. 3, 87-130, DOI 10.4213/im8299; English transl., Izv. Math. 79 (2015), no. 3, 512-553. MR3397413

[26] Yu. A. Neretin, Infinite symmetric groups and combinatorial constructions of topological field theory type (Russian, with Russian summary), Uspekhi Mat. Nauk 70 (2015), no. 4(424), 143204, DOI 10.4213/rm9667; English transl., Russian Math. Surveys 70 (2015), no. 4, 715-773. MR.3400571

[27] Y. A. Neretin, On the group of infinite p-adic matrices with integer elements, Zap. Nauchn. Sem. S.-Peterburg. Otdel. Mat. Inst. Steklov. (POMI) 468 (2018), no. Teoriya Predstavleniı̌, Dinamicheskie Sistemy, Kombinatornye Metody. XXIX, 105-125. MR3869023

[28] G. I. Ol'shanskiu, Unitary representations of $(G, K)$-pairs that are connected with the infinite symmetric group $S(\infty)$ (Russian), Algebra i Analiz 1 (1989), no. 4, 178-209; English transl., Leningrad Math. J. 1 (1990), no. 4, 983-1014. MR.1027466

[29] G. I. Ol'shanskiǔ, Unitary representations of infinite-dimensional pairs $(G, K)$ and the formalism of $R$. Howe, Representation of Lie groups and related topics, Adv. Stud. Contemp. Math., vol. 7, Gordon and Breach, New York, 1990, pp. 269-463. MR1104279

[30] G. I. Ol'shanskiŭ, On semigroups related to infinite-dimensional groups, Topics in representation theory, Adv. Soviet Math., vol. 2, Amer. Math. Soc., Providence, RI, 1991, pp. 67-101, DOI 10.1016/0926-2245(91)90002-q. MR.1104938

[31] Doug Pickrell, Measures on infinite-dimensional Grassmann manifolds, J. Funct. Anal. 70 (1987), no. 2, 323-356, DOI 10.1016/0022-1236(87)90116-9. MR874060

[32] Jean-Pierre Serre, Cours d'arithmétique (French), Collection SUP: "Le Mathématicien", vol. 2, Presses Universitaires de France, Paris, 1970. MR0255476 
[33] Jean-Pierre Serre, Représentations linéaires des groupes finis (French), Hermann, Paris, 1967. MR.0232867

[34] Todor Tsankov, Unitary representations of oligomorphic groups, Geom. Funct. Anal. 22 (2012), no. 2, 528-555, DOI 10.1007/s00039-012-0156-9. MR2929072

[35] André Weil, Basic number theory, Die Grundlehren der mathematischen Wissenschaften, Band 144, Springer-Verlag New York, Inc., New York, 1967. MR0234930

[36] E. I. Zelenov, Representations of commutations relations for p-adic systems of infinitely many degrees of freedom, J. Math. Phys. 33 (1992), no. 1, 178-188, DOI 10.1063/1.529942. MR.1141516

Pauli Institute, Vienna, Austria; Institute for Theoretical Experimental Physics, Moscow, Russia; Department of Mechanics and Mathematics, Moscow State University, Moscow, Russia; and Institute for Information Transmission Problems, Moscow, Russia Current address: Department of Mathematics, University of Vienna, Vienna, Austria $U R L:$ http://mat.univie.ac.at/〜neretin/ 